\title{
Article
}

\section{Smoothed Particle Hydrodynamics (SPH) modelling of transient heat transfer in pulsed laser ablation of Al and associated free-surface problems}

\author{
Al Shaer, Ahmad Wael, Rogers, B.D. and Li, L. \\ Available at http://clok.uclan.ac.uk/18255/ \\ Al Shaer, Ahmad Wael ORCID: 0000-0002-5031-8493, Rogers, B.D. and Li, L. \\ (2017) Smoothed Particle Hydrodynamics (SPH) modelling of transient heat \\ transfer in pulsed laser ablation of Al and associated free-surface problems. \\ Computational Materials Science, 127 . pp. 161-179. ISSN 0927-0256
}

It is advisable to refer to the publisher's version if you intend to cite from the work. http://dx.doi.org/10.1016/j.commatsci.2016.09.004

For more information about UCLan's research in this area go to http://www.uclan.ac.uk/researchgroups/ and search for <name of research Group>.

For information about Research generally at UCLan please go to http://www.uclan.ac.uk/research/

All outputs in CLoK are protected by Intellectual Property Rights law, including Copyright law. Copyright, IPR and Moral Rights for the works on this site are retained by the individual authors and/or other copyright owners. Terms and conditions for use of this material are defined in the policies page. 


\title{
Smoothed Particle Hydrodynamics (SPH) modelling of transient heat transfer in pulsed laser ablation of $\mathrm{Al}$ and associated free-surface problems
}

\author{
A W Alshaer', B D Rogers ${ }^{2}$ and $\mathrm{L} \mathrm{Li}^{1}$ \\ 1 Laser Processing Research Centre, School of Mechanical, Aerospace and Civil Engineering, \\ University of Manchester, M13 9PL, UK \\ ${ }^{2}$ Modelling and Simulation Centre (MaSC), School of Mechanical, Aerospace and Civil Engineering \\ (MACE), University of Manchester, M13 9PL, UK
}

\begin{abstract}
A smoothed particle hydrodynamics (SPH) numerical model is developed to simulate pulsed-laser ablation processes for micro-machining. Heat diffusion behaviour of a specimen under the action of nanosecond pulsed lasers can be described analytically by using complementary error function solutions of second-order differential equations. However, their application is limited to cases without loss of material at the surface. Compared to conventional mesh-based techniques, as a novel meshless simulation method, SPH is ideally suited to applications with highly nonlinear and explosive behaviour in laser ablation. However, little is known about the suitability of using SPH for the modelling of laser-material interactions with multiple phases at the micro scale. The present work investigates SPH modelling of pulsed-laser ablation of aluminium where the laser is applied directly to the free-surface boundary of the specimen. Having first assessed the performance of standard SPH surface treatments for functions commonly used to describe laser heating, the heat conduction behaviour of a new SPH methodology is then evaluated through a number of test cases for single- and multiple-pulse laser heating of aluminium showing excellent agreement when compared with an analytical solution. Simulation of real ablation processes, however, requires the model to capture the removal of material from the surface and its subsequent effects on the laser heating process. Hence, the SPH model for describing the transient behaviour of nanosecond laser ablation is validated with a number of experimental and reference results reported in the literature. The SPH model successfully predicts the material ablation depth profiles over a wide range of laser fluences $4-23 \mathrm{~J} / \mathrm{cm}^{2}$ and pulse durations 6-10 ns, and also predicts the transient behaviour of the ejected material during the laser ablation process. Unlike conventional mesh-based methods, the SPH model was not only able to provide the thermo-physical properties of the ejected particles, but also the effect of the interaction between them as well as the direction and the pattern of the ejection.
\end{abstract}




\section{Keywords}

Smoothed Particles Hydrodynamics (SPH), Heat Conduction, Kernel Correction, Free surface, Laser Ablation, Aluminium.

\section{Introduction}

Lightweight alloys such as aluminium alloys usually have a layer of oxide or hydroxide on their surfaces. These surface layers may deteriorate the properties of the joints after fusion joining due to hydrogen entrapment in the fusion zones [1]. Laser ablation is usually considered to be one of the dry cleaning methods that lead to particles or film ejection from the surface using high-power laser pulses. Ejection of the material from the surface is achieved due to the sudden expansion of the surface particles or by evaporation [2,3]. Laser ablation/cleaning was used as one of the successful applications for weld joint surface preparation for A16014 alloys and Ti6A14V alloys which have been used for automotive and aero-engine manufacture respectively [3, 4].

In order to understand and further improve the process parameters, different modelling approaches have been developed either analytically [5-15], or numerically [13, 16-21]. Conventional numerical modelling methods such as finite elements (FE), finite difference (FD) and finite volume (FV) may not be able to predict complex processes when multiple materials and phases are interacting due to the connected mesh that describes the computational domain. Due to the fact that all the elements in the mesh are connected to each other and cannot leave the mesh, the splashed particles in laser cutting, drilling and ablation cannot be observed in such methods. Some authors [21-23] simulated the protrusion formed by the laser ablation/machining by deleting the elements that have their temperatures greater than the boiling temperature. However, such approach fails to provide information on the behaviour of ejected material. Moreover, FE methods may not be as accurate as required when the studied domain is of micro- or nano-metres scale where the element size should be less than the droplet or the ejected material size by several times [24].

Smoothed Particle Hydrodynamics (SPH) can simulate problems with highly non-linear deformation such as complex movement of multi-phase fluids and topology changes. SPH is one of many meshless (mesh-free) techniques that are based on the Lagrangian description of motion. SPH has proven its ability to model various physical phenomena such as fluid flows, heat and mass transfers, and elasticplastic deformation $[25,26]$. In SPH, the computational domain is divided into arbitrarily distributed points called particles, which move independently from each other eliminating the time and resourceconsuming calculations of spatial derivatives associated with mesh-based methods [27]. SPH was initially developed in 1977 by Lucy L. [28] and Gingold and Monaghan [29] to capture astrophysical phenomena in which boundary conditions are neglected when an open domain is under consideration. Later, by introducing boundary conditions, the SPH method has enabled the simulation of 
engineering problems such as the flow of water waves [30-32], metal forming [33] and phase change problems $[34,35]$. SPH can be used for modelling fusion processes in which liquid or vaporised metals can be handled easily.

SPH modelling of laser processing is still in its early stage and needs significant development since very few (less than ten) publications have been conducted in this field. Demuth et al. [36] simulated laser interference patterning of metallic surfaces using SPH, following Tong and Browne [37] who modelled laser spot welding of aluminium with a very primitive model and limited resolution. Gross's [24] model on laser cutting of metals was further developed by Muhammed et al. [27] who simulated both dry and wet cutting of stainless steel stents used for medical applications, while Yan et al.[38] modelled $\mathrm{CO}_{2}$ laser underwater machining of alumina ceramics. Chen et al. [39] presented their preliminary work on SPH modelling of ultrashort laser pulse interactions with a metal film, in which electron heating and cooling phenomena had to be taken into account. Cao and Shin [40] simulated the phase explosion phenomena in laser ablation of $\mathrm{Al}$ and $\mathrm{Cu}$ at very high laser fluences of up to 36 $\mathrm{J} / \mathrm{cm}^{2}$. The authors presented the ejected material's size distribution during the ablation, but their model did not predict the ablation depth for aluminium at different fluences and did not show the simulation progression to the end of the ablation process although the material's temperature at that time was still above the boiling point and more material was still to be ejected. Hence, the authors did not show the end of the ablation process at which the final ablation depth must be measured and compared with the experiments. Moreover, the proposed SPH model was not able to predict the entire process but relied on molecular dynamics and hydrodynamics models to calculate the initial position of the ejected material, such that the SPH model could not simulate the interaction between the vaporised material and the solid substrate.

Laser material processing depends greatly on the temperature distribution that determines the phase change locus and the change in temperature-dependent properties of the material. None of the previous investigations on SPH modelling of laser heating evaluated the accuracy of temperature values which were mainly used for calculating the melt ejection velocity, kerf width and depth. Cleary \& Monaghan [41] and Jeong et al. [42] studied pure heat conduction problems using SPH and considered the temperature dependence of material properties using a Dirichlet boundary condition. Although the authors investigated different geometries and initial temperature profiles, they only studied the steady-state problem and did not include any heat source terms in their formulation. Additionally, all geometries studied were 2-D closed domains with no treatment of the free-surfaces where the adiabatic condition applies as in laser heating of metal surfaces. Hence, there is a need to investigate the accuracy of transient solutions of problems with rapid heating.

In the present research, thermal conduction of an aluminium sample was investigated using 1-D and 3-D SPH models to evaluate SPH particles' behaviour in laser heating of a metallic free surface. Since 
the temperature distribution follows an error function distribution, the temperature gradient across the sample was compared with the analytical solution and the effect of two kernel correction methods (used to compensate for potential errors at the free surface) were evaluated. Subsequently, a 3-D SPH model is developed to further understand the laser ablation process after comparing the modelling with the analytical solutions in a quasi 1-D model for heat conduction. The results of the simulation were validated with both experimental and numerical data reported in the literature.

\section{Model Description}

\subsection{Physical Phenomena}

\subsubsection{Heat transfer governing equations.}

The mechanism of material removal in laser ablation of metallic alloys will be mainly based on thermal ablation processes when nanosecond laser pulses are used [43]. Figure 1 shows a schematic of a typical laser ablation/cleaning process.

Analytically speaking and taking into account the external heat sources, the differential equation of the heat transfer can be expressed as follows:

$$
\rho c_{p} \frac{d T_{i}}{d t}=\nabla(k \nabla T)+Q-Q_{v}
$$

where $k[\mathrm{~W} / \mathrm{mK}]$ thermal conductivity, $T\left[{ }^{[} \mathrm{K}\right]$ Temperature, $Q\left[\mathrm{~W} / \mathrm{m}^{2}\right]$ the heat source, $Q_{v}$ heat loss due to convection.

The heat loss $Q_{v}$ can be formulated using [27]:

$$
Q_{v}=h_{c}\left(T_{s}-T_{0}\right)+\varepsilon \sigma\left(T_{s}^{4}-T_{0}^{4}\right)
$$

where $h_{c}=20\left[\mathrm{~W} / \mathrm{m}^{2} \mathrm{~K}\right]$ convection factor, $\varepsilon=0.09$ the emissivity, $\sigma=5.67 \times 10^{-8}\left[\mathrm{~W} / \mathrm{m}^{2} \mathrm{~K}^{4}\right]$ the StefanBoltzmann constant, $T_{s}$ and $T_{0}$ are the surface and the initial temperatures respectively. 


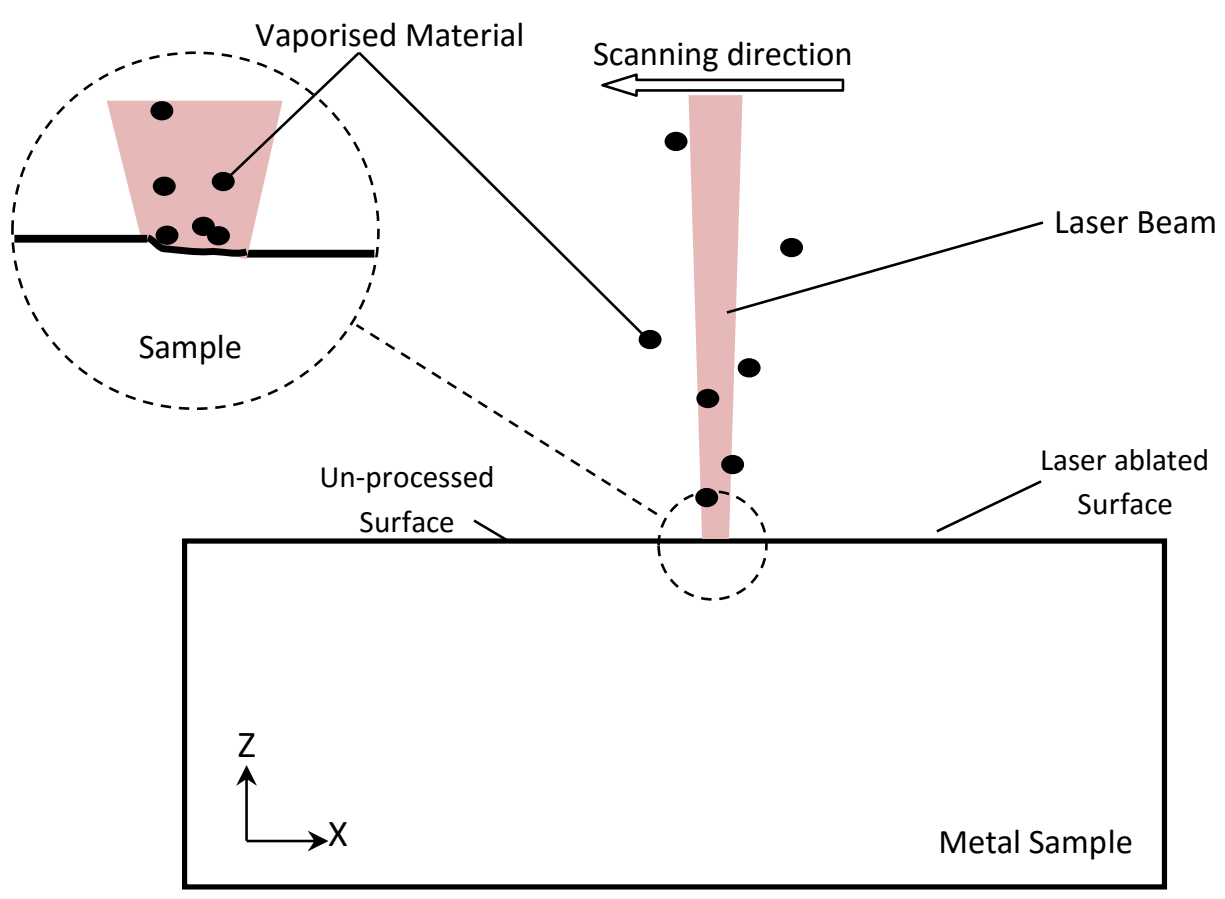

Figure 1 Simplified schematic laser ablation of a metallic sample

The penetration of the electromagnetic wave (laser light) into the material is neglected in this work since the particles' spacing is significantly larger than the optical penetration depth $\left(1 / \alpha_{o}\right)$, where: $\alpha_{o}$ is the optical absorption coefficient for aluminium $\left(1.23 \times 10^{8} \mathrm{~m}^{-1}\right.$ [44]). This means that the electromagnetic wave will be absorbed only by the first layer of particles and the subsequent layers will be mainly heated by conduction

Using Beer-Lambert law, the laser intensity at depth $z$ can be calculated as follows [44] :

$$
I(z)=I_{0} e^{-\alpha_{0} z}
$$

where $I(z)$ is the laser intensity (power per square area) at depth $z, I_{0}$ is the laser intensity at the surface and $z$ is the depth. If $z$ is assumed to be three times the optical penetration $\left(z=3 / \alpha_{o}\right)$, then:

$$
\Rightarrow \frac{I(z)}{I_{0}}=e^{-\alpha_{o} \frac{3}{\alpha_{o}}}=0.049
$$

Hence, the intensity will be reduced to about $5 \%$ of the original intensity within $24.4 \mathrm{~nm}$ from the surface, which is significantly smaller than the particles' spacing used in the simulations. Using the same calculations, the laser intensity at $250 \mathrm{~nm}$ depth will drop to about $0.004 \%$ of the nominal intensity due to the attenuation and the electromagnetic wave will not reach the second layer of particles. Therefore, the optical penetration of the laser beam can be neglected.

\subsubsection{Vapour Pressure.}

The vapour pressure produced by laser ablation of metal can be calculated using the ClausiusClapeyron equation depending on the surface temperature [45]: 


$$
P_{v a p}=P_{a t m} \exp \left(\frac{L_{v}}{R}\left(\frac{T_{s}-T_{b}}{T_{S} \cdot T_{b}}\right)\right)
$$

where $P_{a t m}, L_{v}, R, T_{b}$ and $T_{s}$ are atmospheric pressure, latent heat of vaporisation, the gas constant, the boiling temperature and the surface temperature respectively.

\subsubsection{Vapour velocity.}

Assuming that the vapour particles are expelled from the surface with a one-dimensional Maxwellian velocity, the vapour velocity can be approximated using the average velocity in the normal direction at temperature $T_{s}$ as follows [46]:

$$
v_{v a p, s}=\sqrt{\frac{2 k_{B} T_{s}}{\pi m}}
$$

where $k_{B}$ is Boltzmann constant $\left(1.38 \times 10^{-23} \mathrm{~m}^{2} \mathrm{~kg} / \mathrm{s}^{2} \mathrm{~K}\right)$, and $m$ is the atomic mass. It should be noted that this equation gives a good approximation when the experiments are conducted in a vacuum or when the vapour pressure is much greater than the surrounding pressure.

\section{SPH Methodology}

\subsection{SPH Interpolation}

In SPH, the computational domain is divided into arbitrarily distributed particles where each has its unique properties including mass $m_{\mathrm{i}}$, volume $\omega_{\mathrm{i}}$, pressure $P_{\mathrm{i}}$, and velocity $v_{\mathrm{i}}$ [47]. The value of a function $A(\boldsymbol{r})$ at location $\boldsymbol{r}$ can be found by a local interpolation for a set of surrounding particles at a specific time step. In continuous form, the interpolated value of the function can be estimated [25]:

$$
\langle A(\boldsymbol{r})\rangle=\int_{\Omega} W\left(\boldsymbol{r}-\boldsymbol{r}^{\prime}, h\right) A\left(\boldsymbol{r}^{\prime}\right) d \Omega
$$

where $\boldsymbol{r}$ is a position vector, $<>$ denotes approximation, $W$ is the weighting function called the smoothing kernel, $h$ is the smoothing length (a characteristic length scale of the kernel). For the interaction between two SPH particles $i$ and $j$ the smoothing kernel can be written in a general form as follows [48]:

$$
W_{i j}=W\left(\boldsymbol{r}_{\boldsymbol{i} \boldsymbol{j}}, h\right)=\frac{1}{h^{n}} f\left(\frac{\left|\boldsymbol{r}_{\boldsymbol{i}}-\boldsymbol{r}_{\boldsymbol{j}}\right|}{h}\right)
$$

where $n$ is the number of spatial dimensions, $f$ is a function of $h$ and $r_{i j}=\left|\boldsymbol{r}_{i}-\boldsymbol{r}_{j}\right|$ the distance between two particles $i$ and $j$.

The function $A(\boldsymbol{r})$ can be written in discrete SPH form as follows: 


$$
\left\langle A_{i}(\boldsymbol{r})\right\rangle=\sum_{j} m_{j} \frac{A_{j}}{\rho_{j}} W_{i j}
$$

where $m_{j}$ and $\rho_{j}$ are the particles mass and density respectively.

Accordingly, the gradient of the considered function can be calculated by taking the kernel gradient into account in the approximation:

$$
\left\langle\nabla A_{i}(\boldsymbol{r})\right\rangle=\sum_{j} m_{j} \frac{A_{j}-A_{i}}{\rho_{j}} \nabla_{i} W_{i j}
$$

To conserve momentum in $\mathrm{SPH}$, a slightly different form is used to calculate the pressure gradient as shown later in equation (21):

$$
\frac{\left\langle\nabla A_{i}(\boldsymbol{r})\right\rangle}{\rho_{i}}=\sum_{j} m_{j}\left(\frac{A_{i}}{\rho_{i}^{2}}+\frac{A_{j}}{\rho_{j}^{2}}\right) \nabla_{i} W_{i j}
$$

Equation (10-1) is used for divergence operators, such as in the conservation of mass equation introduced below, while Equation (10-2) is used to ensure an equal and opposite reaction between particles as in the case of calculating the pressure gradient. The latter formula conserves linear and angular momentum (for further information see Ref. [25, 49])

The smoothing kernel can take different forms; Gaussian, Quadratic, cubic spline (B-spline), or higher order kernels fourth \& fifth order. The cubic spline was selected to be used in our model since it approximates the Gaussian function and is commonly used throughout SPH. The cubic spline can be expressed by the following equation [25]:

$$
W\left(r_{i j}, h\right)=\alpha_{D}\left\{\begin{array}{cc}
1-\frac{3}{2} q^{2}+\frac{3}{4} q^{3} & 0 \leq q \leq 1 \\
\frac{1}{4}(2-q)^{3} & 1 \leq q \leq 2 \\
0 & 2 \geq q
\end{array}\right.
$$

where $q=r_{i j} / h, \alpha_{\mathrm{D}}$ is a normalization parameter to ensure the unity integral of the kernel, with a value of $2 h / 3$ for 1 -D model and $1 /\left(\pi^{2} / 3 . h^{3}\right)$ for 3-D. Figure 2 shows particle $i$ and its neighbour particles $j$ within a smoothing kernel of a radius $2 h$. 


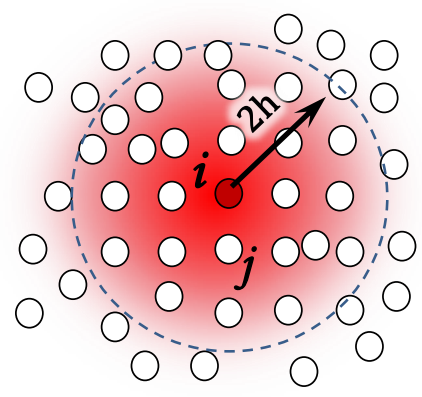

Figure 2 Particles $i$ and $j$ within the smoothing kernel support.

\subsection{Kernel gradient correction}

The kernel shown in Figure 2 is said to have complete support since the considered particle, $i$, is surrounded by particles which contribute to its interpolation during the simulation. However, there are cases where the kernel support is incomplete such as when the particle approaches the boundaries or when the particle is at a free surface. Different measures have been introduced to SPH to compensate for such loss of support [50-52], such as kernel gradient correction KGC [53] and the Schwaiger operator [54] that were developed to correct the variables' gradients and the second derivatives respectively. This is potentially important for laser processing where the specimen is heated by the laser acting on a free surface with the temperature evolution controlled by thermal diffusion which is described mathematically using a second-order diffusion term (Equation 1).

The kernel gradient correction method replaces the normal kernel gradient $\nabla_{i} W_{i j}$ by a corrected kernel gradient $\widetilde{\nabla}_{i} W_{i j}$ expressed using the following set of equations [55]:

$$
\begin{gathered}
\widetilde{\nabla}_{i} W_{i j}=L_{i} \nabla_{i} W_{i j} \\
L_{i}=M_{i}^{-1} \\
M_{i}=\sum_{j}^{n u m} \frac{m_{j}}{\rho_{j}} \nabla_{i} W_{i j} \otimes\left(\boldsymbol{x}_{\boldsymbol{i}}-\boldsymbol{x}_{\boldsymbol{j}}\right)
\end{gathered}
$$

The corrected gradient is therefore given by:

$$
\left\langle\nabla A_{i}(\boldsymbol{r})\right\rangle^{K G C}=\sum_{j} m_{j} \frac{A_{j}-A_{i}}{\rho_{j}} \widetilde{\nabla}_{i} W_{i j}
$$

To correct the values of the second derivative, a modified Laplacian operator should be used along with the corrected kernel gradient according to the following approximation [54]: 


$$
(\nabla . \mu \nabla f)_{i}=\frac{\operatorname{tr}(\Gamma)^{-1}}{n}\left\{\sum_{j} \frac{m_{j}}{\rho_{j}}\left(\mu_{i}+\mu_{j}\right)\left(\frac{f_{i}-f_{j}}{r_{i j}^{2}}\right) \boldsymbol{r}_{i j} \cdot \widetilde{\nabla}_{\imath} W_{i j}-\left[\nabla\left(\mu_{i} f_{i}\right)-f_{i} \nabla \mu_{i}+\mu_{i} \nabla f_{i}\right] .\left(\sum_{j} \frac{m_{j}}{\rho_{j}} \widetilde{\nabla_{l}} W_{i j}\right)\right\}
$$

where $\mu$ is a physical constant such as a diffusion coefficient or thermal conductivity, $n$ is the number of dimensions, $f$ is the value of the function, and $\Gamma$ is a tensor:

$$
\Gamma_{\beta \gamma}=\sum \frac{\boldsymbol{r}_{i j} \cdot \nabla W_{i j}}{r_{i j}^{2}} \Delta x_{\beta} \Delta x_{\gamma}
$$

where subscripts $\beta, \gamma$ denote the computational domain directions.

As known, the second derivative (Laplacian) of a temperature is an essential quantity in the heat conduction equation (1) to calculate the rate of change in temperature over time, and hence to calculate temperature values at a specific time.

\subsection{Governing Equations and SPH Discretisation}

SPH uses a Lagrangian formulation for the SPH particles moving in space and time. In this work, the specimen will be modelled as a viscous fluid. The Lagrangian form of conservation of momentum and continuity equations for fluids can be expressed from the general form of Navier-Stokes equations as:

$$
\begin{gathered}
\frac{D \boldsymbol{v}}{D t}=-\frac{1}{\rho} \nabla P+v \cdot \nabla^{2} \boldsymbol{v}+\boldsymbol{g} \\
\frac{D \rho}{D t}=-\rho \nabla \cdot \boldsymbol{v}
\end{gathered}
$$

where $\boldsymbol{v}$ is the velocity, $P$ is pressure, $v$ is viscosity and $\boldsymbol{g}=(0,0,-9.81) \mathrm{ms}^{-2}$ is the gravitational force.

The previous equations (18 and 19) can be discretized in SPH as [56]:

$$
\begin{gathered}
\frac{d \rho_{i}}{d t}=-\rho_{j} \sum_{j} \frac{m_{j}}{\rho_{j}} \boldsymbol{v}_{\boldsymbol{i j}} \cdot \nabla_{i} W_{i j} \\
\frac{d \boldsymbol{v}_{\boldsymbol{i}}}{d t}=-\sum_{j} m_{j}\left(\frac{P_{i}+P_{j}}{\rho_{i} \rho_{j}}\right) \nabla_{i} W_{i j}
\end{gathered}
$$

where $\boldsymbol{v}_{i j}=\boldsymbol{v}_{i} \boldsymbol{v}_{j}$. Note that Equation (21) uses the anti-symmetric form of the gradient (Equation 102) to conserve momentum.

In this work, the viscosity introduced by Monaghan [56] was used to eliminate any unphysical instability in the model [57]. These forces were expressed by inserting artificial viscosity into the momentum equation to become: 


$$
\frac{d \boldsymbol{v}_{\boldsymbol{i}}}{d t}=-\sum_{j} m_{j}\left(\frac{P_{i}+P_{j}}{\rho_{i} \rho_{j}}+\prod_{i j}\right) \nabla_{i} W_{i j}+\boldsymbol{g}
$$

where $\prod_{i j}$ is the artificial viscosity:

$$
\begin{gathered}
\prod_{i j}=\left\{\begin{array}{cc}
\frac{-\alpha c_{i j} \mu_{i j}}{\rho_{i j}} & \boldsymbol{v}_{\boldsymbol{i j}} \cdot \boldsymbol{r}_{\boldsymbol{i j}}>0 \\
0 & \boldsymbol{v}_{\boldsymbol{i j}} \cdot \boldsymbol{r}_{\boldsymbol{i j}}<0
\end{array}\right. \\
\mu_{i j}=\frac{h \boldsymbol{v}_{\boldsymbol{i j}} \cdot \boldsymbol{r}_{\boldsymbol{i j}}}{r_{i j}{ }^{2}+\eta_{i j}^{2}} \\
c_{i j}=\frac{1}{2}\left(c_{i}+c_{j}\right) \quad, \quad \rho_{i j}=\frac{1}{2}\left(\rho_{i}+\rho_{j}\right)
\end{gathered}
$$

In order to simplify the model, $\alpha$ was selected " 5.0 " to simulate the solid phase as a high viscous liquid, and " 0.1 " for the liquid phase.

Additionally, the pressure in this model was expressed by Tait's equation of state [58]:

$$
P=B\left[\left(\frac{\rho}{\rho_{0}}\right)^{\gamma}-1\right]
$$

where $B=\frac{\rho_{0} c_{0}^{2}}{\gamma}, \rho_{0}=1000 \mathrm{~kg} / \mathrm{m}^{3}$ is the reference density, $c_{0}$ speed of sound, and $\gamma=7$ is a constant.

To simulate the thermal behaviour of particles, an SPH form of the heat conduction equation (1) was introduced into the model to include the laser beam heating of the surface [58]:

$$
c_{p} \frac{d T_{i}}{d t}=\sum_{j} \frac{m_{j}}{\rho_{i} \rho_{j}}\left(\frac{4 k_{i} k_{j}}{k_{i}+k_{j}}\right)\left(\frac{T_{i}-T_{j}}{r_{i j}^{2}}\right) \boldsymbol{r}_{i j} \nabla_{i} W_{i j}+Q-Q_{v}
$$

In laser ablation processes such as laser cleaning, the laser beam acts only on the free surface of the sample and the attenuation of the electromagnetic field into the material is neglected as discussed in section 2.1.1.

In SPH, the location of free surface can be determined by computing the divergence of the particle position using the following equation [47]:

$$
\nabla . r=\sum_{j} \frac{m_{j}}{\rho_{j}} \boldsymbol{r}_{i j} . \nabla W_{i j}
$$

The truncated kernel support of any surface particle gives a non-zero value for the particle position divergence. In 3-D cases, an empirical value $(\nabla . r<2.4)$ was used to indicate a free-surface particle $[59,60]$. 


\subsection{Time-step scheme and CFL number}

The Predictor-Corrector scheme was used in this work to evaluate the parameters over time. In this scheme, the variables values are calculated in time as follows [55]:

$$
\begin{gathered}
\boldsymbol{v}_{\boldsymbol{i}}^{n+1 / 2}=\boldsymbol{v}_{\boldsymbol{i}}^{n}+\frac{\Delta t}{2} \boldsymbol{F}_{i}^{n} ; \rho_{i}^{n+1 / 2}=\rho_{i}^{n}+\frac{\Delta t}{2} D_{i}^{n} \\
\boldsymbol{r}_{\boldsymbol{i}}^{n+\mathbf{1} / \mathbf{2}}=\boldsymbol{r}_{i}^{n}+\frac{\Delta t}{2} \boldsymbol{V}_{i}^{n} ; e_{i}^{n+1 / 2}=e_{i}^{n}+\frac{\Delta t}{2} E_{i}^{n} \\
T_{i}^{n+1 / 2}=T_{i}^{n}+\frac{\Delta t}{2} T_{i}^{n}
\end{gathered}
$$

where $n$ superscript denotes the current time step.

The values are then corrected at the half step and finally calculated at the end of the time step as follows:

$$
\left.\begin{array}{c}
\boldsymbol{v}_{i}^{n+1}=2 v_{i}^{n+1 / 2}-v_{i}^{n} \quad ; \quad \rho_{i}^{n+1}=2 \rho_{i}^{n+1 / 2}-\rho_{i}^{n} \\
\boldsymbol{r}_{i}^{n+1}=2 \boldsymbol{r}_{i}^{n+1 / 2}-\boldsymbol{r}_{i}^{n} ; \quad e_{i}^{n+1}=2 e_{i}^{n+\frac{1}{2}}-e_{i}^{n} \\
T_{i}^{n+1}=2 T_{i}^{n+1 / 2}-T_{i}^{n}
\end{array}\right\}
$$

Usually, a variable time step is selected to replace the constant time step when some physical variables vary drastically over time. This requires a change in time-step according to CFL (CourantFriedrich-Lewy) condition, the forcing terms, the viscous diffusion term [50] and importantly for the application presented herein the thermal diffusion term [58]. The variable time step can be calculated according to:

$$
\begin{gathered}
\Delta t=C F L \cdot \min \left(\Delta t_{f}, \Delta t_{c v}, \Delta t_{D}\right) ; \Delta t_{f}=\min \left(\sqrt{\frac{h}{\left|f_{i}\right|}}\right) ; \Delta t_{c v}=\min _{i}\left(\frac{h}{c_{s}+\max _{j}\left|\frac{h v_{i j} \boldsymbol{r}_{i j} \mid}{r_{i j}^{2}}\right|}\right) \\
\Delta t_{D}=\frac{\rho c_{p} \Delta x^{2}}{k}
\end{gathered}
$$

where $\Delta t_{f}$ is based on the specific force, $\Delta t_{c v}$ combines Courant and the viscous terms and $\Delta t_{D}$ is based on the thermal diffusion term.

\subsection{Boundary Conditions}

The model utilises dynamic boundary conditions in which the boundaries consist of three layers of particles arranged in a staggered position. The boundary particles were created to be fixed fluid particles with zero velocity to simulate the solid walls surrounding the studied sample. The 
conservation equations are calculated for all particles including the boundary and this provides the interactions between the moving and the boundary particles. More details can be found in Ref. [61].

\section{Results and Discussion}

The laser ablation process involves repeated cycles of heating and cooling. For simple cases (without ablation or phase change), these heating and cooling processes can be modelled analytically (1-D) when the temperature distribution is known to be characterised by an error function [62].

Before simulating the laser ablation process using a 3-D model, several steps have to be completed in order to validate the results obtained from this model. As mentioned earlier, the SPH interpolation procedure has incomplete support at or near a surface which can introduce numerical errors. Firstly, a preliminary study was carried out to determine whether or not any kernel corrections were needed at the sample surface where the kernel support was incomplete. Secondly, a convergence study was performed to specify the required resolution and the time step for the simulation.

\subsection{Kernel Gradient Correction (KGC)}

Using a 1-D SPH model, two test cases were performed to investigate the performance of the SPH model by evaluating the errors in the functions' gradient and the second derivative at the free surface for functions encountered in heating. The gradient and the second derivative were calculated for six different functions: three polynomial functions (first, second and third order), hyperbolic, logarithmic and complementary error functions. Since the temperature distribution for a surface heat source follows an error function, this is of particular interest herein. The ordinary SPH results with no corrections were examined and compared to the same cases with the Kernel Gradient Correction (KGC) and Schwaiger operator correction. Figure 3 shows the different functions plotted for a variable $x=[1,5] \mathrm{m}$, using a particle spacing of $0.25 \mathrm{~m}$, and $h=1.5 \Delta x$. The main aim of testing various functions with/without correction is to observe the SPH model behaviour and sensitivity to approximating functions of different orders, especially interpolating the error functions that describe the temperature evolution during laser heating of metals. The analytical values of the gradient and Laplacian can be easily obtained by differentiating the six functions once and twice respectively. 


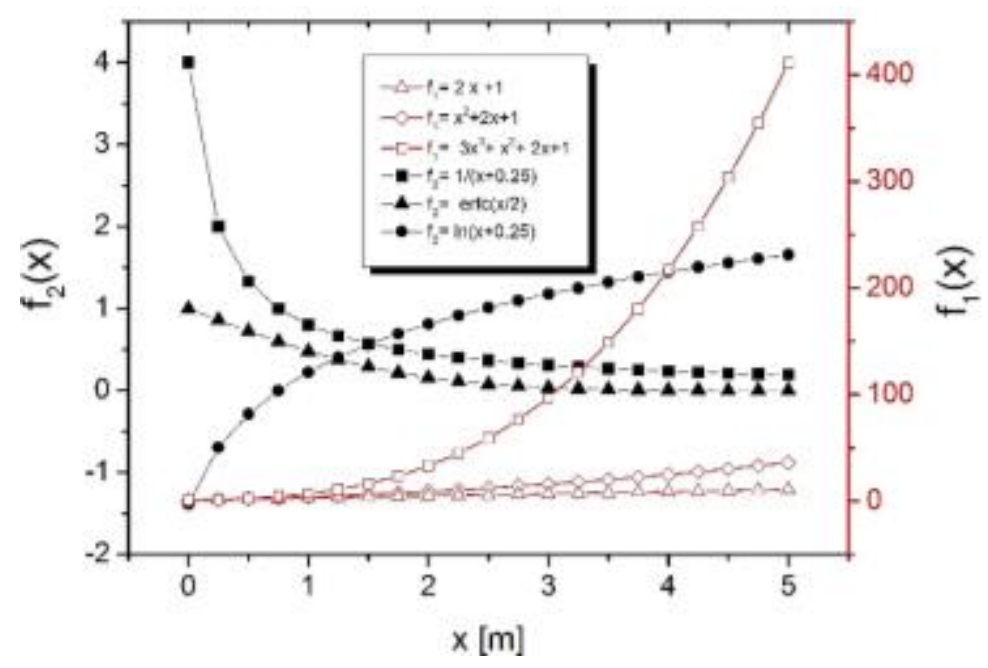

Figure 3 Functions used to validate the kernel truncation correction at the free surface in SPH

Figure 4 depicts the values of the functions' gradient across the sample calculated using an uncorrected SPH gradient (Equation 10) and a corrected kernel gradient (Equation 15) both numerically and analytically. It is pertinent to mention that with $h=1.5 \Delta x$, the kernel support was truncated over three layers of particles starting from each end of the domain corresponding to $2 h=3 \Delta x$ distance.

In order to quantify the error generated during the simulations, the L2 norm error [63], that is used to evaluate the discrepancy in the SPH results from the analytical solution, can be calculated as follows:

$$
L_{2}(\Phi)=\sqrt{\sum_{i=1}^{N}\left(\Phi^{s p h}-\Phi^{\text {theory }}\right)^{2}}
$$

where $\phi^{\text {sph }}$ is SPH temperature value, $\phi^{\text {theory }}$ is the analytical value and $N$ is the number of particles.

As expected for the uncorrected SPH, Figure 4 shows that the agreement is satisfactory away from the surface, but poor within $2 h$ of the surface where the kernel support is incomplete. Whereas, the kernel gradient correction succeeds in compensating for the loss of support at the boundaries and produces a very good correlation with the analytical solution for the polynomial and the error functions. This can also be seen in Figure 5 which shows that KGC eliminated the error for the linear and quadratic functions and reduced the error for the cubic and the error functions significantly. However, the influence of KGC on the logarithmic and hyperbolic functions' gradient was not as significant as on the other functions since their numerical and analytical results are still far from each other at the boundaries. 

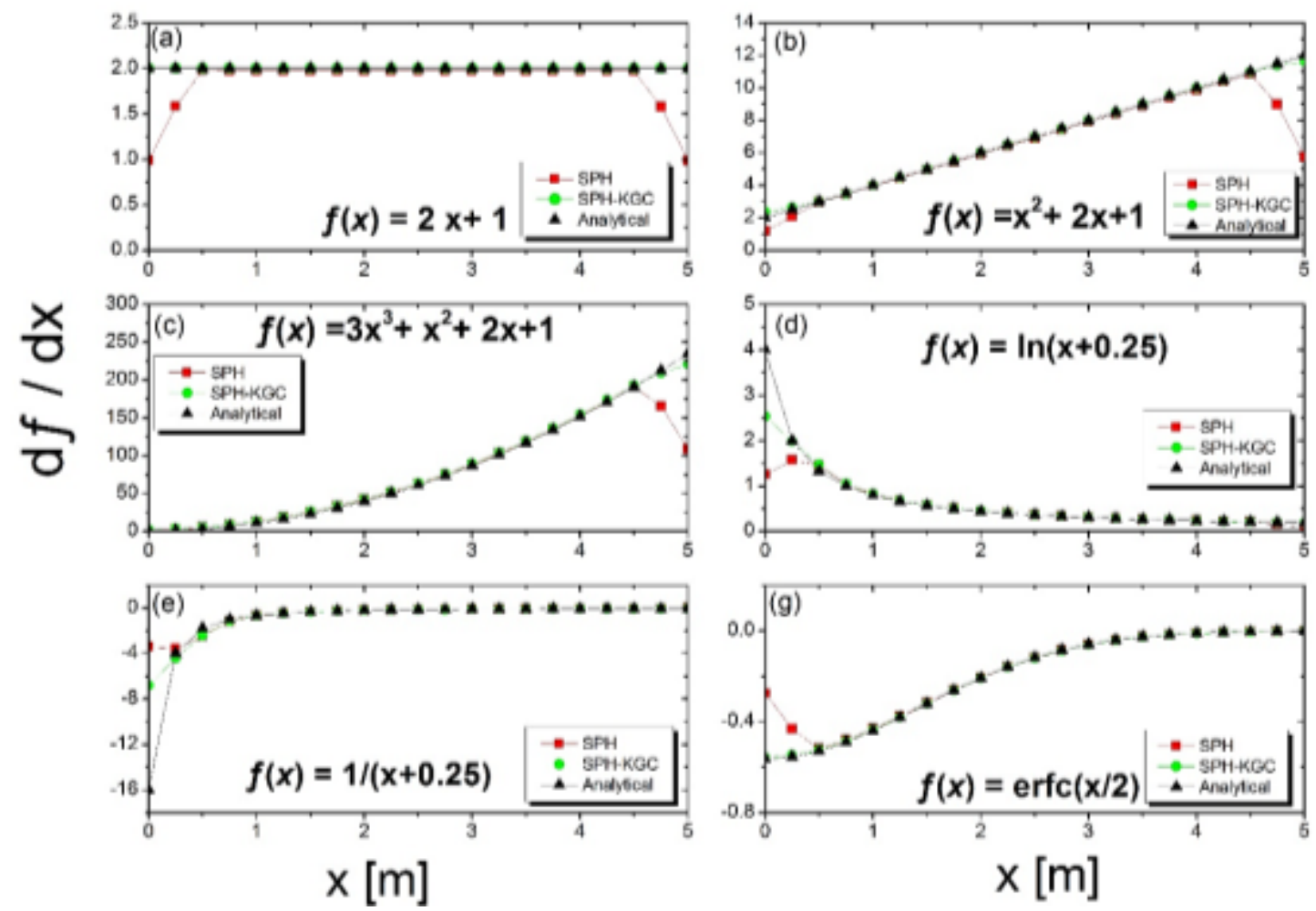

Figure 4 Kernel gradient Correction (KGC) effect on SPH results at the free ends of the 1-D domain Although KGC was not able to eliminate the error for the logarithmic and hyperbolic functions entirely, it managed to reproduce the analytical solution for particles located at $x=0.5 \mathrm{~m}$ achieving a gradient profile in closer agreement with the analytical solution than the one produced without correction.

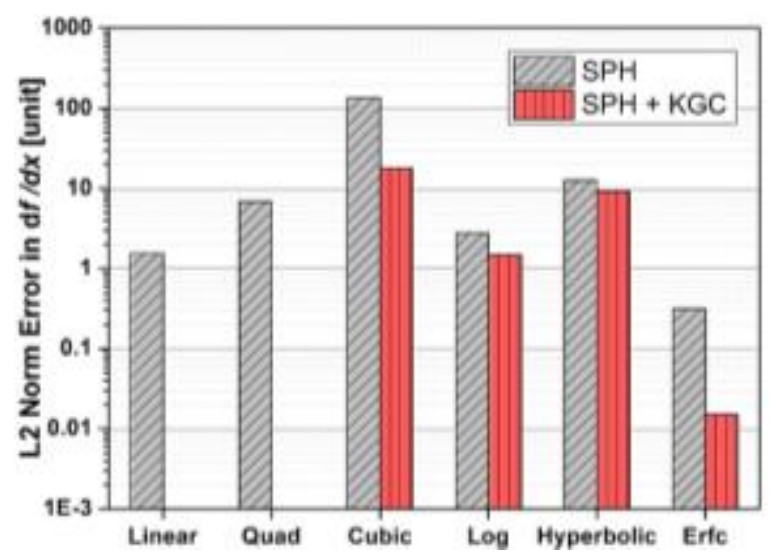

Figure 5 L2 Norm Errors in SPH results for the gradient of different functions with/without Kernel Gradient Correction

Generally, the difference between SPH with/without correction results should clearly appear at each end of the computational domain that suffers from a lack of support. However, the values of the gradients in some cases (Figure $4 \mathrm{c}, \mathrm{d}$, e and g) tend to zero at one end due to the nature of the function, making the results of SPH with/without KGC difficult to distinguish. Therefore, it is 
sufficient to evaluate the efficiency of the correction method only at one end where the difference in the results is evident.

According to the aforementioned discussion, the kernel gradient correction may be a suitable method for correcting the gradients of physical parameters that follow linear or error functions at the free surfaces such as thermal heating and cooling during laser processing of metals.

\subsection{Laplacian Operator Correction (Schwaiger correction)}

Since the thermal behaviour is described by a second-order derivative (Equation 1) which in SPH requires a combined use of two first-order operators (Equation 27), another test case was conducted to examine the effect of the Schwaiger operator with/without the KGC on the second derivative of the same functions. Figure 6 and Figurer 7 show the SPH results of the functions' second derivative without and with KGC respectively, while Figure 8 depicts the L2 Norm errors produced in each test case.
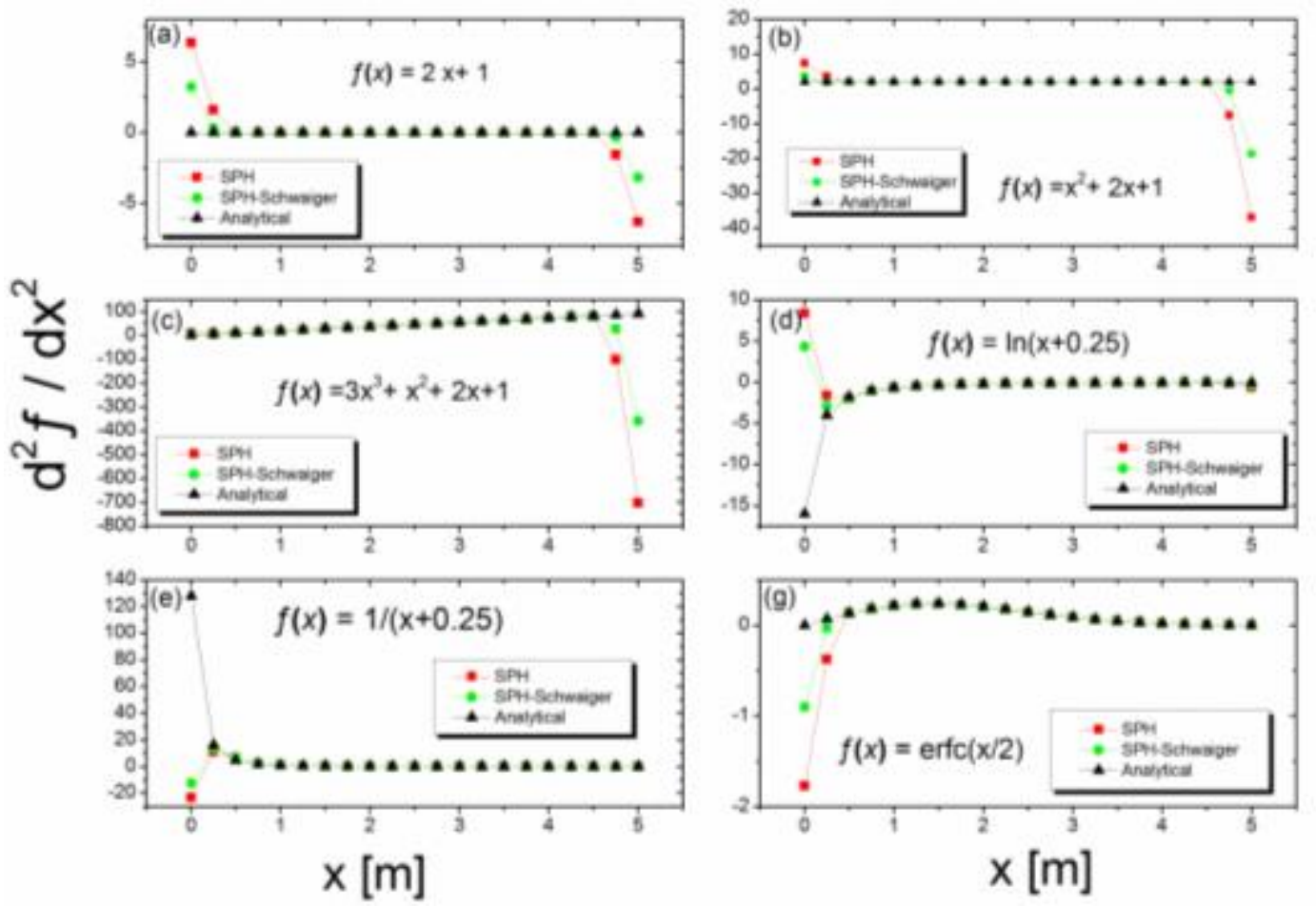

Figure 6 Effect of Schwaiger operator without KGC on SPH results at the boundaries of the 1-D domain

Figure 6 clearly shows that without KGC, the standard SPH second-order derivative performs poorly near a free surface and the Schwaiger operator helps to reduce the error but not eliminate it.

It can be seen from Figure 8 that Schwaiger operator without KGC has reduced the errors in the numerical results by about $10 \%$ to $60 \%$ for all different functions. However, the second derivative correction had less impact on the logarithmic and hyperbolic functions than on the other four 
functions. This can be attributed to the large difference between $d^{2} f / d x^{2}$ values in the first and the second particles caused by the nature of the functions' derivatives since they tend to infinity when $x$ value tends to zero. This, therefore, makes the drastic change in $d^{2} f / d x^{2}$ difficult to be captured using SPH formulations without further corrections. Moreover, it is already known $[59,60]$ that the kernel gradient correction should be coupled with the Schwaiger operator in order to obtain satisfactory results for the second derivative.

After applying Schwaiger correction with KGC, Figure 7 shows that the second-order derivative values improved significantly with a closer agreement with the analytical solution for all functions. This can be justified in Figure 8 which shows that the error for the linear function was eliminated and the errors were reduced by about $70 \%$ to $90 \%$ for the quadratic, cubic and the error functions. However, less impact on the errors for the logarithmic and hyperbolic functions can be observed with only $10 \%$ to $15 \%$ reduction in comparison with Schwaiger correction without KGC.

From Figures 6, 7 and 8, it is important to note that this method of correction achieved a very good correlation with the analytical solution for the "Erfc" function type that can be used to describe the temperature change during laser heating of metals. However, any changes in the formulation of the Erfc function given in Figure $7 \mathrm{~g}$ ) require an evaluation as to whether or not a Schwaiger correction is needed. This comes from the fact that the function formulation has a significant impact on the functions behaviour in space and time. The sign of the second-order derivative depends on the positive direction of the axis of concern in Figures 6 and 7. However, the bottom end of the substrate in the 3-D simulation is supported by the boundary particles limiting the kernel truncation problem to the free top surface. The large jump in temperature can be modified by selecting the correct particle spacing and time step (which was done in Sec 4.3.2) and the appropriate correction method (KGC, Schwaiger) if needed. However, since the surface temperature is effectively specified by the external heat source, $Q$, on particles that would otherwise have required these corrections, this obviates the need for corrections. This matter is thoroughly discussed in Sec 4.3.2." 

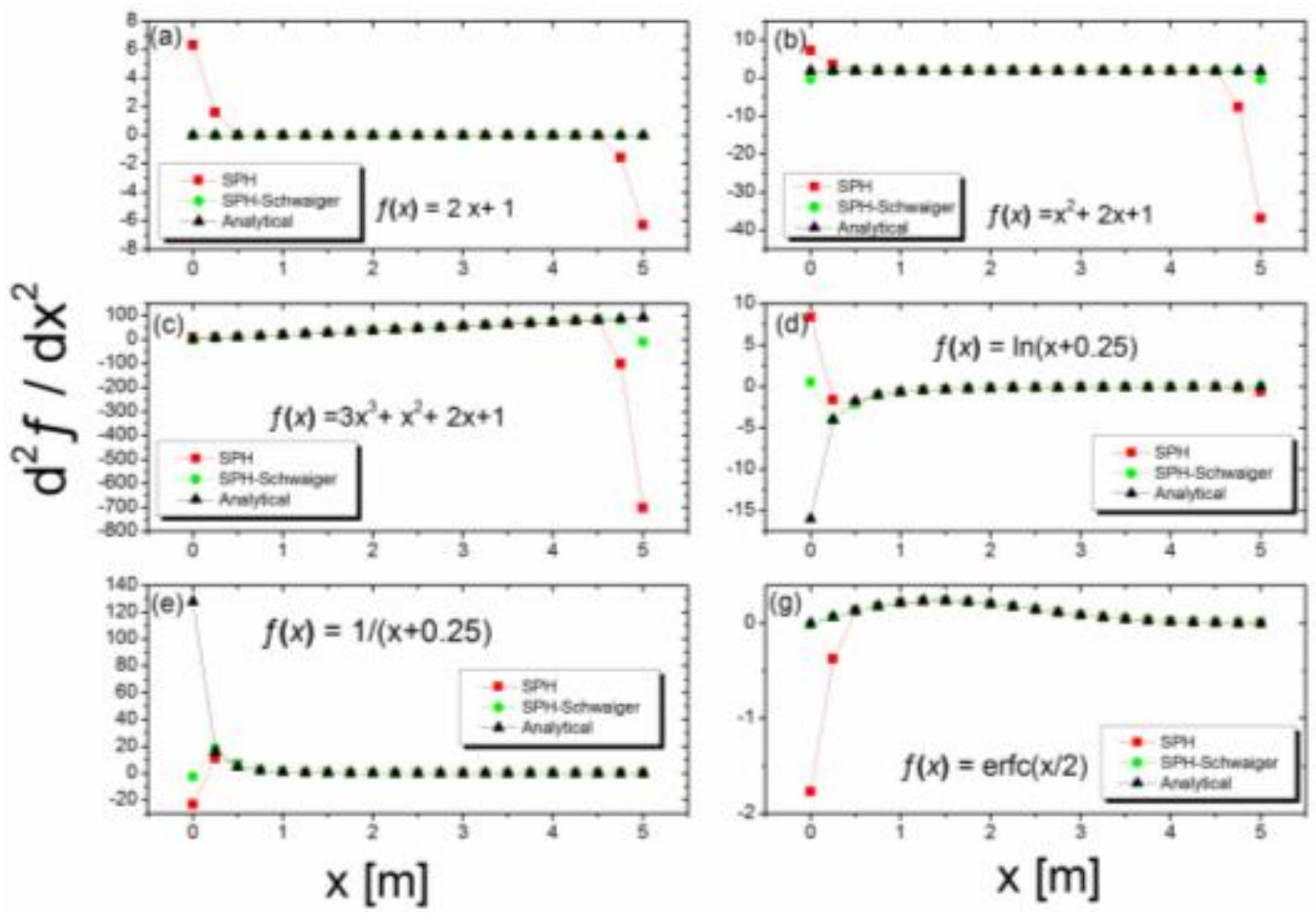

Figure 7 Effect of Schwaiger operator with KGC on SPH results at boundaries of the 1-D domain

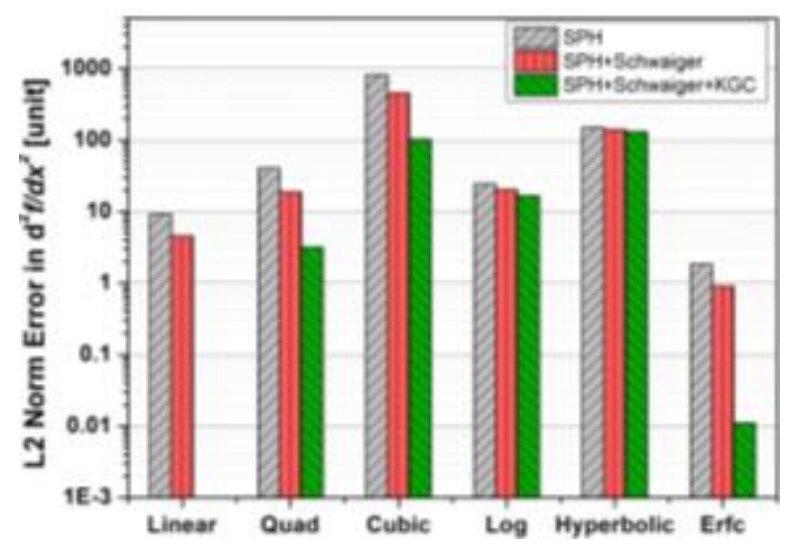

Figure $8 \mathrm{~L} 2$ Norm Errors in SPH results for the second derivative of different functions with/without correction

To conclude, the combination of the KGC and Schwaiger corrections was able to correct the errors caused by the truncated support at the domain borders for the gradient and Laplacian of the studied functions respectively. However, for non-linear functions such as hyperbolic and logarithmic functions both correction methods were only able to reduce the deviation at the boundaries without matching the analytical solution. Nevertheless, these methods are essential for correcting the gradient and the second derivatives of the polynomial and the error functions. 
Therefore, it is recommended in SPH modelling to take into account the nature of the functions that describe the physical phenomena, especially when modelling free surfaces at which the kernel support is incomplete.

\subsection{Transient Heat Transfer Test Cases}

\subsubsection{Numerical setup.}

In this work, a 3-D SPH model of $20 \times 20 \times 200 \mu \mathrm{m}^{3}$ was created with $0.2 \mu \mathrm{m}$ spacing and a free surface on the top to simulate pulsed laser ablation of aluminium and its alloys (see Figure 1). At the beginning of the simulation, the sample was assumed to be at room temperature. The model was created using a modified SPHysics [55] serial code and was run using an Intel ${ }^{\circledR}$ Core i7 CPU (3.4 $\mathrm{GHz}$ ) with 8 GB RAM on an Ubuntu 14.4 LTS operating system.

Additionally, a 1-D SPH code was compiled using Matlab 2014a and the numerical results were compared with the analytical solution of the 1-D heat transfer partial differential equation (PDE). The 1-D analytical solution of the heat conduction PDE for multi-pulses is given in Appendix A.

The pulsed laser was simulated during the validation studies using $100 \mathrm{~ns}$ pulse duration (laser-ON), $100 \mathrm{~ns}$ relaxation time (laser-OFF) and a $9.6 \times 10^{11} \mathrm{~W} / \mathrm{m}^{2}$ laser intensity. Two different reflectivity values were selected to cover the different surface conditions that may be encountered when ablating aluminium alloys. Pure aluminium or polished aluminium alloys surfaces have a very high reflectivity of about $95 \%$, while oxidised, contaminated or coated aluminium surfaces have lower reflectivity reaching up to 75\% [3]. The thermo-physical properties of the base material are listed in Table 1 and are assumed to be temperature-independent during the simulations.

Table 1 Aluminium alloy AA6014 thermo-physical properties [64]

\begin{tabular}{ccccccc}
\hline $\begin{array}{c}\text { Density } \\
\rho\left[\mathrm{kg} / \mathrm{m}^{3}\right]\end{array}$ & $\begin{array}{c}\text { Surface Optical } \\
\text { Reflectivity } \\
R[\%]\end{array}$ & $\begin{array}{c}\text { Thermal } \\
\text { diffusivity } \\
D\left[\mathrm{~m}^{2} / \mathrm{s}\right]\end{array}$ & $\begin{array}{c}\text { Initial } \\
\text { Temperature } \\
{\left[{ }^{0} \mathrm{~K}\right]}\end{array}$ & $\begin{array}{c}\text { Thermal Conductivity } \\
k\left[\mathrm{~W} / \mathrm{m} .{ }^{0} \mathrm{~K}\right]\end{array}$ & $\begin{array}{c}\text { Specific Heat } \\
C_{p}[\mathrm{~J} / \mathrm{kg}]\end{array}$ & Emissivity \\
\hline 2705 & $75 \%$ and $95 \%$ & $6.89 \times 10^{-5}$ & 300 & 167 & 896 & 0.09 \\
\hline
\end{tabular}




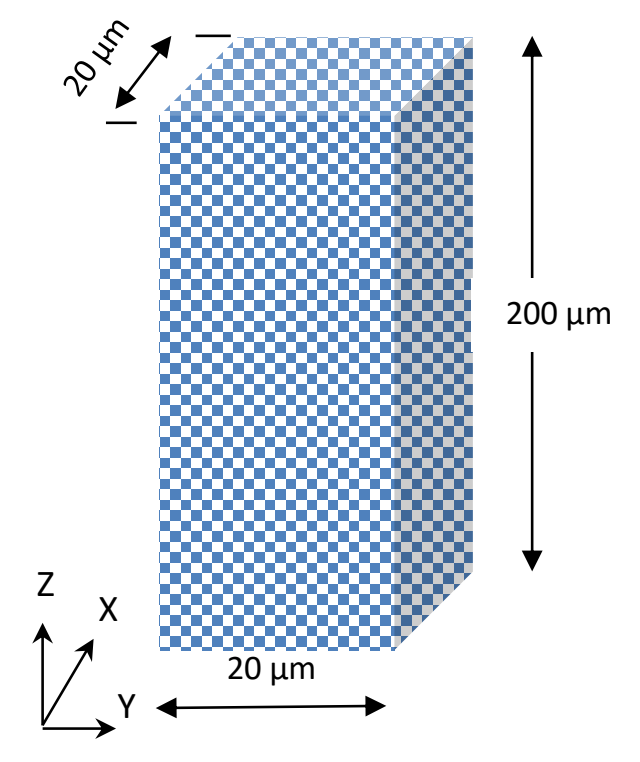

Figure 9 SPH computational domain (not to scale)

\subsubsection{Convergence Studies.}

In laser-metallic interactions, the laser beam can only act on the metallic surface and the heat dissipates into the sample to the lower layers by heat conduction. The laser removes, melts or evaporates the material from the surface. Accordingly, gaining the correct temperature values at the surface is essential in understanding the laser thermal ablation processes. With the results for different functions in section 4.2, a test case was conducted to examine the temperature values at the surface of a metallic sample where the laser beam heating is active over typical pulse duration of $100 \mathrm{~ns}$.

As mentioned previously in sections 4.1 and 4.2, the effect of corrections is dependent on the type and the behaviour of the function being evaluated at the surface. The error function that was used in sections 4.1 and 4.2 was only a function of $x$ and did not vary with time. Therefore, error function cases that are dependent on variables including time will show a different response to the truncation of the kernel at the surface and hence their values should also be evaluated against the corresponding analytical solution through new test cases.

The investigation in Section 4.2 showed that both kernel gradient correction and the Schwaiger correction are necessary to obtain satisfactory agreement with the analytical result for typical functions describing heat transfer, for example in the form of a complementary error function. For surface laser application, since the surface temperature is effectively specified by the external heat source, $Q$, on particles that would otherwise have required these corrections, this obviates the need for both the kernel gradient and Schwaiger corrections. Moreover, Figures 6a, b, d and g show that the second layer of particles has errors, but when the temperature of the surface particles is determined by 
the applied laser, the error at the second layer of particles is also reduced as will be demonstrated in the temperature profiles presented in section 4.3.3.3). For the analytical cases presented herein for pulsed lasers with heat loss, during the very short laser-off period (100 ns), the surface particles transfer heat to interior particles only which do not require the corrections. This case is different from the internal (volume) heating in which the heat diffuses from the inside of the domain towards the free surface at which the thermal boundary conditions and the kernel support will be the main factor to determine the temperature values. As a result, no corrections are applied in the laser ablation model.

The particles were kept stationary during the simulation to observe the heat transfer behaviour of the solid particles and the temperature produced at the surface. It should be noted that the model dimensions were selected to enhance the heat flow in one direction (z-direction) in order for the results to be validated with a separate SPH 1-D code results. A 3-D model will represent an aluminium rod being heated at one end and will be referred to as "quasi 1-D" model. After validation, the dimensions will be changed to reflect the 3-D aspects of the problems in the real applications as will be presented in the "laser ablation model" section of this paper.

First, two convergence studies were conducted to determine the correct resolution (particles' spacing) and the time step. To determine the initial particles' spacing, an initial value of $5 \mathrm{~ns}$ for the time step was selected to calculate the thermal penetration caused by pulsed laser heating of the surface:

$$
z=\sqrt{D \cdot t_{p}}=\sqrt{0.689 \times 10^{-4} \times 5 \times 10^{-9}} \approx 0.6 \mu \mathrm{m}
$$

where $D$ is thermal diffusivity, $t_{p}$ is laser pulse duration and $z$ is thermal penetration depth.

Equation (34) shows that the heat wave will travel $0.6 \mu \mathrm{m}$ inside the sample within $5 \mathrm{~ns}$ of the heating time. Hence, a value of $1 \mu \mathrm{m}$ (that is larger than $0.6 \mu \mathrm{m}$ ) was chosen as an initial particle spacing to start the convergence study for the selected model. The initial value of $1 \mu \mathrm{m}$ was selected to be of the same order of the calculated thermal penetration depth and to generate an integer number of particles (20 particles) along the smallest dimension in the computational domain, namely $20 \mu \mathrm{m}$. To examine numerical convergence, three different resolutions were used: $1 \mu \mathrm{m}, 0.5 \mu \mathrm{m}$ and $0.25 \mu \mathrm{m}$ respectively.

Figure 10 shows the temperature evolution on the top surface using three different resolutions for a single-pulse laser ablation, and using the analytical solution as given in Appendix A. It is important to note that some of the literature on pulsed laser beam heating used these equations to describe the temperature during multi-pulses heating by only replacing $T_{0}$ with the temperature value from the preceding pulse. This treatment is incorrect due to the different boundary conditions associated with equations (A.1) and (A.2) which are different from the conditions applied in multi-pulses heating i.e. the temperature profile across the sample after the first pulse is not identical to the constant temperature distribution across the sample at the beginning of the process. Therefore, the temperature 
increase produced by each laser pulse should count for all preceding heating and cooling cycles of the preceding pulses. Hence, the number of heating and cooling terms in the previous equations will change accordingly for each pulse.

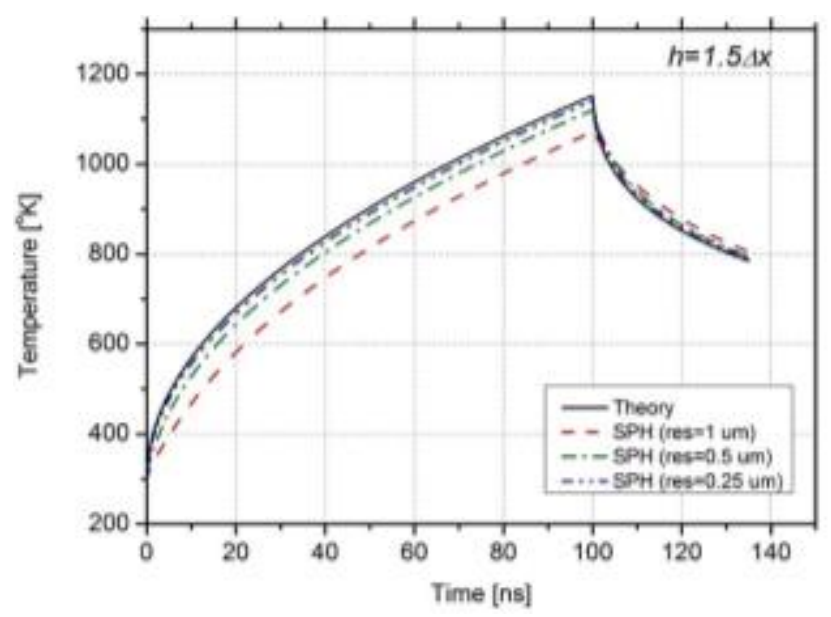

Figure 10 Surface temperature using different particles spacing for single Laser pulse $t_{p}=100 \mathrm{~ns}$.

It can be seen from Figure 10 that reducing the particle spacing from $1 \mu \mathrm{m}$ to $0.25 \mu \mathrm{m}$ led to smaller deviations of the SPH results with the analytical solution. This can be attributed to the increase in the number of particles within the thermal penetration depth ( $\sim 3$ particles at $0.25 \mu \mathrm{m}$ spacing). Herein, to quantify the rate of convergence, the errors are quantified using the $L_{2}$ error norm since a uniform particle refinement ratio is used.

Figure 11 shows that reducing the spacing from $1 \mu \mathrm{m}$ to $0.25 \mu \mathrm{m}$ decreased the error in temperature by approximately $85 \%$ from $20 \mathrm{~K}$ to only $3 \mathrm{~K}$. Moreover, plotting the $L_{2}$ error norm in Figure 11 indicates a first order convergence which is consistent with the order of convergence calculated using the expression suggested by Roache [65] for a three-resolution system with constant refinement ratio $(r)$ :

$$
P=\frac{\log \left(\frac{f_{3}-f_{2}}{f_{2}-f_{1}}\right)}{\log (r)}=1.12
$$

where $f_{3}, f_{2}$, and $f_{l}$ are the values of the temperature using the finest resolution to coarsest resolution.

Taking into account the exact temperature values and the numerical results for the finest resolution, the relative error and the Grid Convergence Index (GCI) for this case can be calculated from:

$$
\varepsilon=\frac{f_{1}-f_{\text {exact }}}{f_{\text {exact }}}=-0.0086
$$




$$
G C I_{21}=F_{s} \frac{|\varepsilon|}{\left(r^{P}-1\right)}=0.019
$$

where $F_{s}$ is a safety factor taken as " 1.25 " and is based on experience by applying GCI in different applications [66].

The value of GCI indicates that the SPH results lie within a $1.9 \%$ deviation band from the exact solution with a $95 \%$ confidence level.

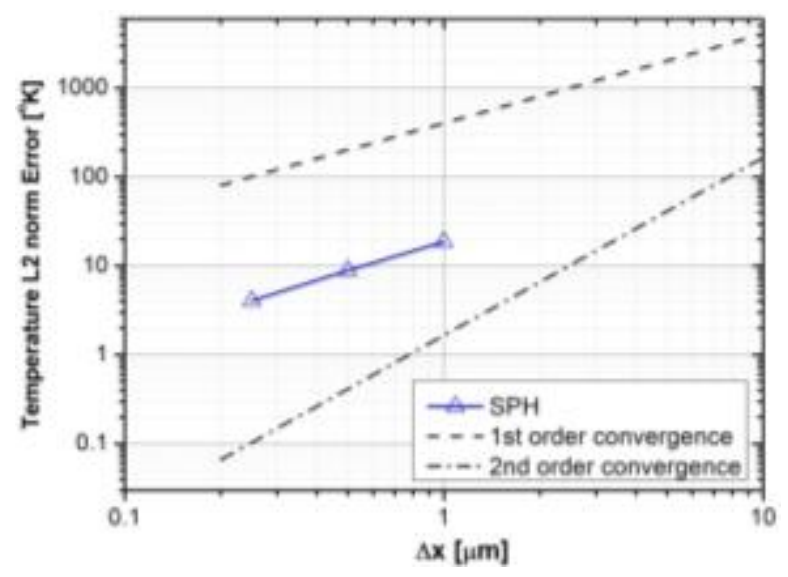

Figure 11 L2 Norm Error for SPH results at different resolutions

In order to obtain good simulation results over time, the time step should be selected to capture all parameters' changes during the simulation, without increasing the CPU time at no gain. It can be seen from the analytical solution in Figure 10 that the temperature changes sharply at the beginning of the heating and cooling phases by about $150-200 \mathrm{~K}$ within 3-4 nanoseconds i.e. about $50 \mathrm{~K} / \mathrm{ns}$. Using the material properties and the numerical parameters, the set of equations (32) show that the largest time step to be used in the simulation should be $0.9 \mathrm{~ns}$ in order to capture the sharp change in temperature.

Figure 12 shows that the use of $0.5 \mathrm{~ns}$ time step with CFL number of 0.1 enables the simulation to capture the drastic change in the surface temperature, especially at the beginning of both the heating and the cooling phases. However, the selection of longer time steps such as $1 \mathrm{~ns}$ and $5 \mathrm{~ns}$ destabilises the simulation and terminates it at the beginning. 


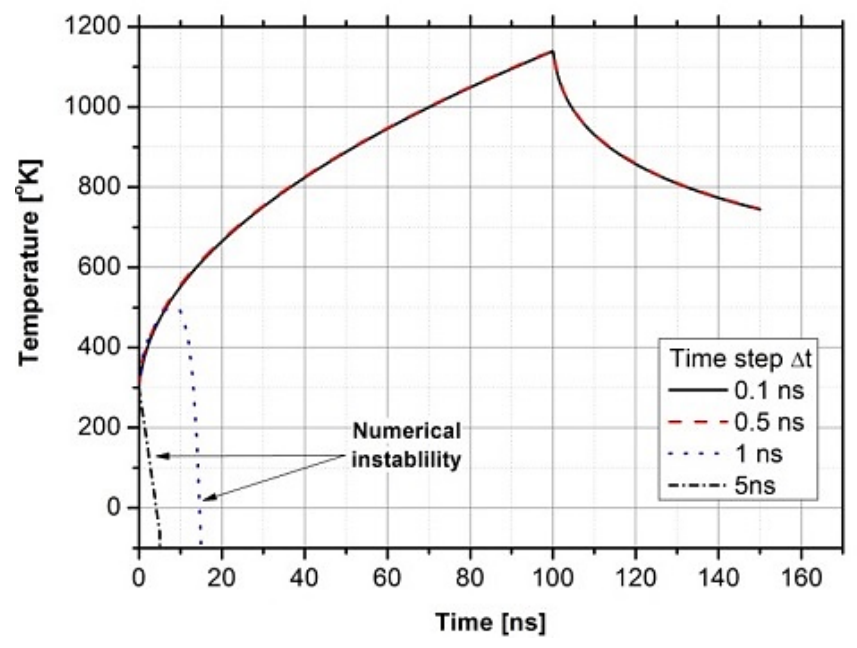

Figure 12 Effect of time step in SPH modelling using the step-predictor corrector time scheme

\subsubsection{Pulsed Laser Transient Heating.}

\subsubsection{Single Heating and Cooling Cycle.}

The ability of SPH to predict the cooling effect once the heat source is removed was assessed by simulating a single pulse of 100 ns duration and a relaxation time up to $8 \mu$ s as illustrated in Figure 13. These temporal values correspond to a $20 \mathrm{kHz}$ pulse frequency, which is commonly used in laser ablation processes as will be demonstrated in the following sections. The 3-D model predicted the heating and cooling of the aluminium sample precisely over time after the surface temperature reached more than $4500 \mathrm{~K}$ at the end of the pulse. It is pertinent to mention that laser pulses heat and cool the material rapidly as shown in Figure 13 in which the temperature dropped to less than the melting point within less than $2 \mu \mathrm{s}$. As will be discussed in the following sections, rapid heating and cooling are very beneficial in metal laser ablation because it leads to a smaller heat affected zone HAZ and less distortions. 


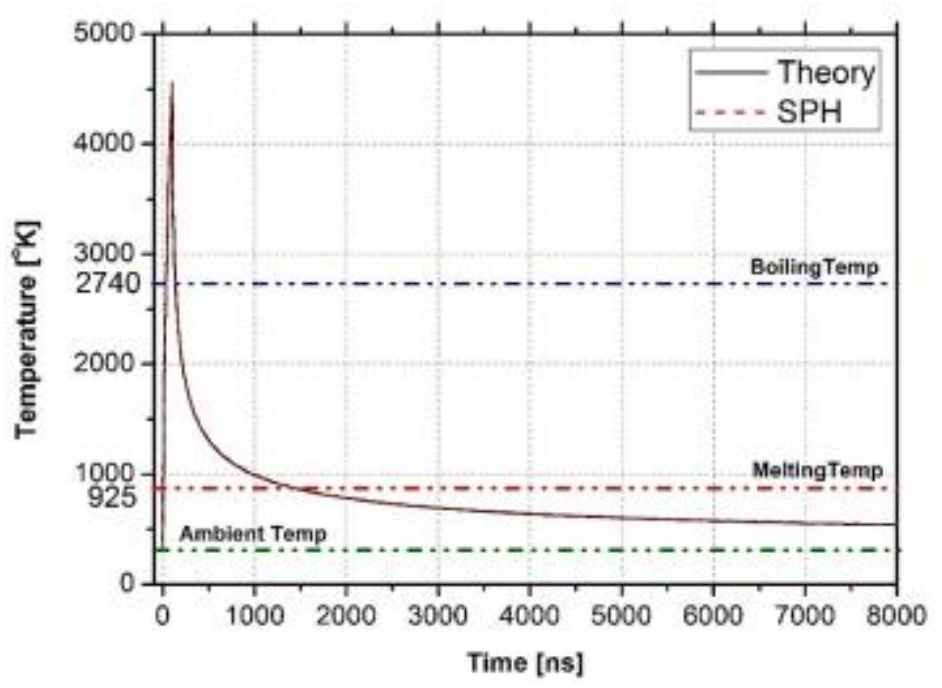

Figure 13 Single Laser pulse of $100 \mathrm{~ns}$ and $75 \%$ surface reflectivity on the aluminium target using the 3-D SPH code.

\subsubsection{Multiple Heating and Cooling Cycles: Surface Temperature.}

A second stage of validating the SPH model is to make a comparison between the temperature evolution over time between the 1-D and the quasi-1D models comparing with an analytical solution for cyclic heating. Figure 14 shows the surface temperature change due to pulsed laser heating using two reflectivity values (a) 95\%, and (b) 75\%, in which equal heating and relaxation periods were selected to observe the effect of multiple pulses within a short time of laser heating. The correct temperature distribution during the frequent pulses heating will generate the correct crater depth, ejected material's characteristics and its behaviour.

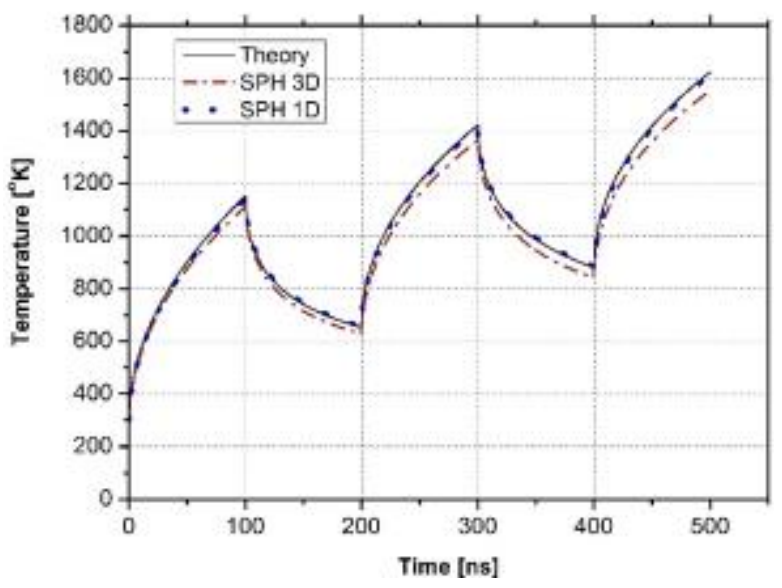

(a)

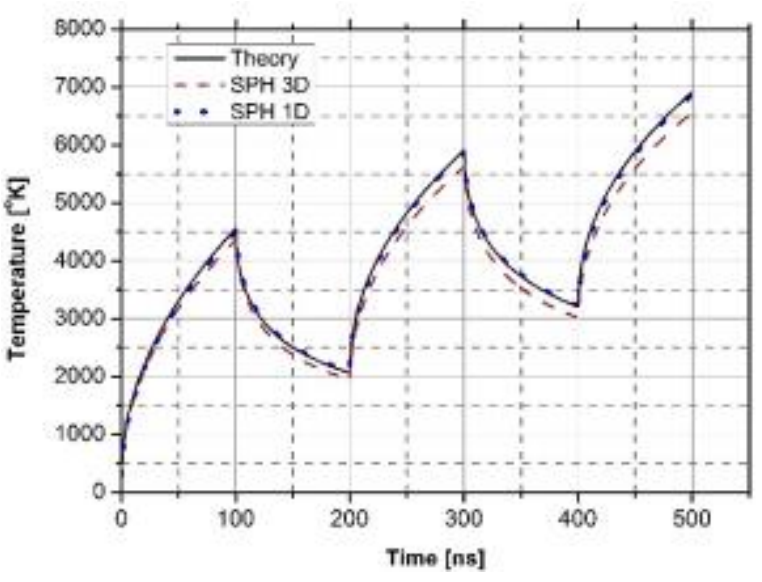

(b)

Figure 14 SPH modelling of three consecutive laser pulses of $100 \mathrm{~ns}$ pulse duration and $100 \mathrm{~ns}$ relaxation time (a) 95\% surface reflectivity (b) 75\% surface reflectivity 
From Figure 14, the 1-D model predicts the surface temperature with only $0.8 \%$ error for both high and low reflectivity, while the 3-D model showed a slight deviation from the theory by about $2.5 \%$ in the peak temperature at the end of each heating stage. This small difference between the two models can be attributed to the existence of the dynamic boundary (DB) particles on the sides of the computational domain in the 3-D model. These particles were kept at the room temperature as would occur in the real applications since this model will be later modified to simulate the laser ablation process. These boundary particles slightly cool the adjacent particles by conduction causing the surface temperature to drop by about $2.5 \%$ in comparison with the analytical value. The DB particles existence is very important to imitate the solid aluminium medium that surrounds either molten or vaporised matter that will be seen in the proceeding sections of this paper. Although some of the accuracy is sacrificed by introducing the DB particles, their physical significance justifies the need for them.

\subsubsection{Multiple Heating and Cooling Cycles: Temperature Distributions.}

Figure 15 shows (a) the temporal variation in temperature at different depths from the sample surface, and (b) the temperature profile across the sample over time. Additionally, Figure 16 (a) and (b) plot the time derivative of temperature, $\mathrm{d} T / \mathrm{d} t$ (that is the heating or cooling rates), at the surface and at different depths respectively. During all heating phases in Figure 15 (a), it is evident that the surface temperature climbs rapidly to more than $800^{\circ} \mathrm{K}$ within $20-40 \mathrm{~ns}$ at the beginning of the pulse, which corresponds to a very high heating rate $\left(\sim 6 \times 10^{10} \mathrm{~K} / \mathrm{s}\right)$ distinguished by the positive value shown in Figure 16 (a). At the start of the pulse, an instant high value of the heating rate appears immediately due to the instant application of the laser pulse. After each occasion that the laser is turned off, the heating rate reduces over time due to the heat being conducted to the lower layers (shown at depths of $5 \mu \mathrm{m}$ and $7 \mu \mathrm{m}$ in Figure 15(a)) whose temperatures gradually increase, reducing the difference in the surface temperature.
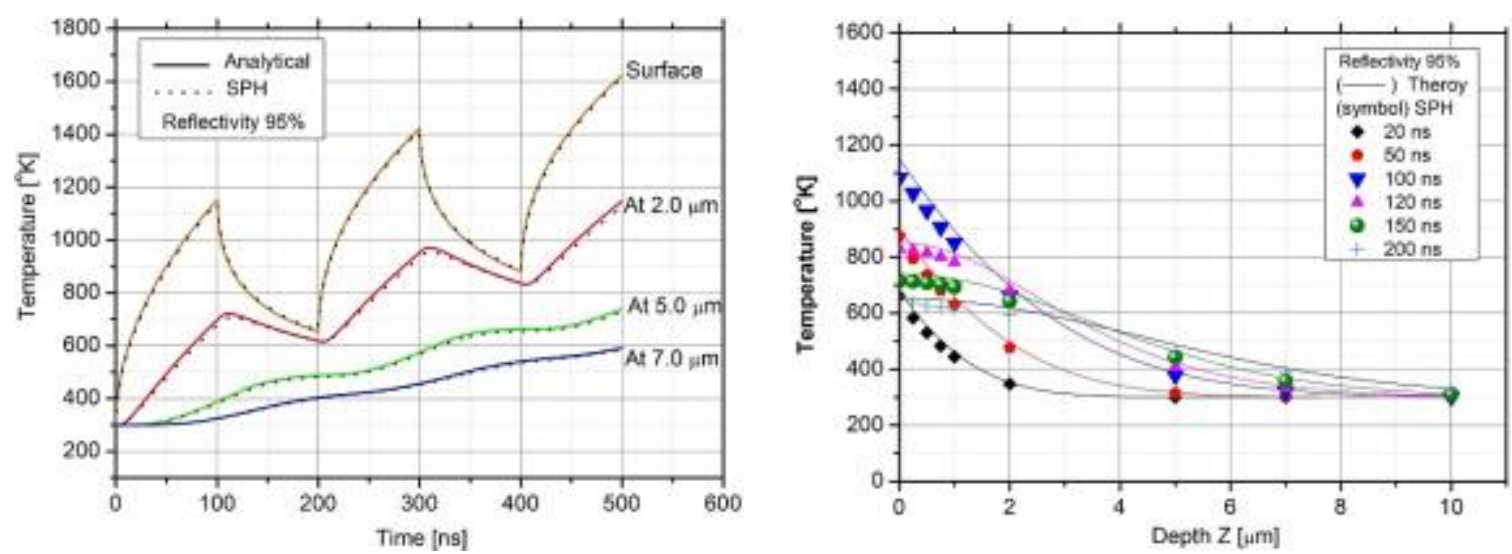

Figure 15 Temperature history during laser heating (a) Temperature variation over time (b) Temperature profile across the sample 

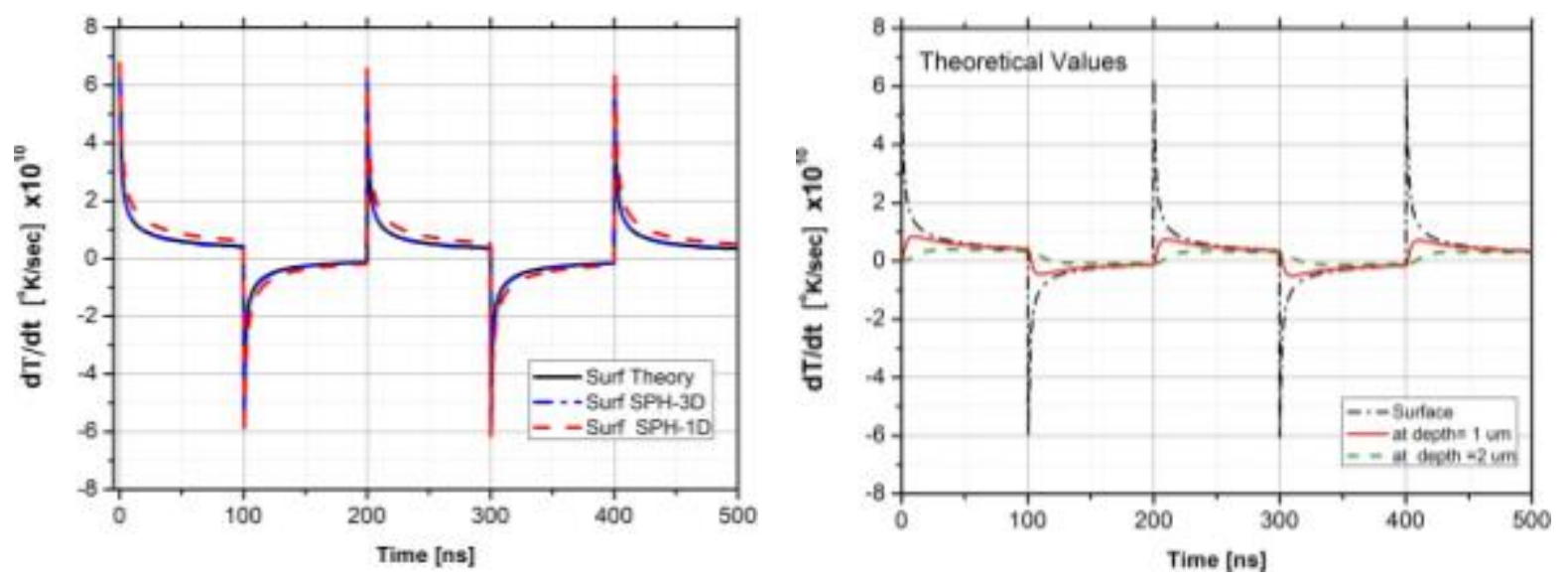

Figure 16 Heating/Cooling rates at the surface during three consecutive laser pulses

At the beginning of the cooling phases $(t=100,300,500, \ldots \mathrm{ns}), \mathrm{d} T / \mathrm{d} t$ becomes instantaneously negative indicating that only cooling is taking place at the surface and therefore the surface of the sample is transferring the heat rapidly to the lower layers without gaining or losing heat from or to any external sources. The cooling rate then reduces with time since the lower layers' temperature is tending to the surface temperature to achieve thermal equilibrium. This is very clear from Figure 16 (a) where $\mathrm{d} T / \mathrm{d} t$ is tending to zero at the end of each cooling phase (at $200 \mathrm{~ns}$ and $400 \mathrm{~ns}$ ) and in Figure 15 (a) the temperature at $2 \mu \mathrm{m}$ depth is tending towards the temperature of the surface. Moreover, it should be recognised that the peak temperature at $2 \mu \mathrm{m}, 5 \mu \mathrm{m}$, and $7 \mu \mathrm{m}$ are delayed relative the surface temperature by time shifts of 10-60 ns as shown in Figure 15 (a). This is due to the time needed for the heat wave to propagate into the sample body, which is dependent on the thermal conductivity and other thermal properties of the base material. Additionally, this peak also depends on the depth at which the temperature is calculated, i.e. the deeper the layer the greater the time by which the peak is shifted.

\subsection{Laser Ablation Model}

With the satisfactory agreement of the SPH solution with a 1-D analytical solution, the model is now applied to laser ablation cases. In order to evaluate the performance of the SPH model for laser ablation prediction, different test results were compared against published data on laser ablation of aluminium. To simulate the material ablation, the boiling temperature of aluminium $\left(2730^{\circ} \mathrm{K}\right)$ was set as a thermal criterion to eject the SPH particles from the surface (equation 27) assuming that a small portion of the heat delivered by the laser is being wasted due to convection and radiation. It is important to mention that the fluences used in those studies lies within the low to medium fluence ranges in comparison with the high regime (order of $10^{3} \mathrm{~J} / \mathrm{cm}^{2}$ ) in which the phase explosion ${ }^{1}$ [67] is the predominant mechanism in the process. The surface temperature at high fluences may exceed the

\footnotetext{
${ }^{1}$ Phase explosion occurs when the material's temperature exceeds the thermodynamic critical temperature $T_{\text {tc }}$ and a large amount of nuclei starts to form at a homogenous rate in a very short time
} 
critical temperature for aluminium ( 8000 K [68]), at which the vapour phase volume breaks down and starts interacting with the incident laser beam. This range of fluences is beyond the scope of this paper.

It can be noted that the ablated surface approximates the shape of a flat plane following the same spatial distribution of the laser pulse (Top-Hat). If a Gaussian pulse is used, a more bell-like shape can be seen (see Figure 17) due to the concentrated energy at the centre rather than at the circumference.

\subsubsection{Ablation depth.}

The process parameters used in Lutey's et al. [69] experimental work were introduced into the SPH model as given in Table 2. Running the simulation for $15 \mathrm{~ns}$ using $0.2 \mu \mathrm{m}$ particle spacing, Figure 18 shows the temporal progression of the ejected material as well as the temperature profile across the sample within the active beam zone. It is pertinent to mention that a Top-Hat beam profile and a square laser pulse were used during the simulation to reproduce the experimental conditions.

Table 2 Material properties and process parameters used in the SPH model

\begin{tabular}{ccccccccc}
\hline Material & $\begin{array}{c}\text { Density } \\
\rho \\
{\left[\mathrm{kg} / \mathrm{m}^{3}\right]}\end{array}$ & $\begin{array}{c}\text { Initial } \\
\text { Temperature } \\
{\left[{ }^{0} \mathrm{~K}\right]}\end{array}$ & $\begin{array}{c}\text { Thermal } \\
\text { Conductivity } \\
{\left[\mathrm{W} / \mathrm{m} .{ }^{0} \mathrm{~K}\right]}\end{array}$ & $\begin{array}{c}\text { Specific } \\
\text { Heat } \\
{[\mathrm{J} / \mathrm{kg}]}\end{array}$ & $\begin{array}{c}\text { Pulse } \\
\text { duration } \\
{[\mathrm{ns}]}\end{array}$ & $\begin{array}{c}\text { Fluence } \\
{\left[\mathrm{J} / \mathrm{cm}^{2}\right]}\end{array}$ & $\begin{array}{c}\text { Repetition } \\
\text { rate } \\
{[\mathrm{kHz}]}\end{array}$ & $\begin{array}{c}\text { Simulation } \\
\text { timestep } \\
{[\mathrm{ns}]}\end{array}$ \\
\hline Aluminium & 2705 & 300 & 167 & 896 & 10 & 10 & 30 & 0.05 \\
\hline
\end{tabular}

The laser pulse was activated instantly at the beginning of the simulation and was deactivated at $10 \mathrm{~ns}$ leaving the top surface to cool naturally due to the conducted heat to the rest of the bulk material. Due to the very high irradiance $\left(1 \mathrm{GW} / \mathrm{cm}^{2}\right)$ acting on the top surface, the temperature of the surface exceeded the boiling temperature of aluminium within only $1 \mathrm{~ns}$, reaching about $3744 \mathrm{~K}$.

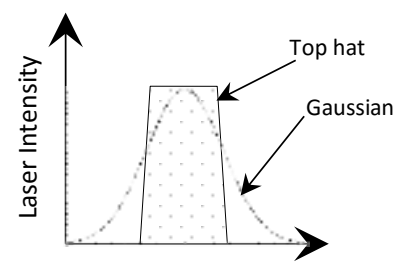

Figure 17 Spatial distribution of the Laser intensity as a function of time

Once the surface particles are ejected in reality, they lose the interaction with the laser beam (apart from particle scattering) allowing the laser to heat up the newly exposed layer. Therefore, it is assumed that there will be no interaction with the laser beam once particles abandon the surface. Hoffman and Szymasnki [70] calculated the optical penetration for different metallic vapours at different temperatures. For aluminium vapour with a temperature less than $4000 \mathrm{~K}$, the calculated 
absorption coefficient was about $4 \times 10^{-2} \mathrm{~m}^{-1}$ which corresponds to $25 \mathrm{~nm}$ optical penetration at $10 \mu \mathrm{m}$ laser wavelength. For shorter wavelengths such as $1.064 \mu \mathrm{m}$, the optical penetration will be even higher. Using the calculated optical penetration, the aluminium vapour at distances $2 h=6 \times 10^{-7} \mathrm{~m}$ from the surface will absorb only $2.4 \times 10^{-6} \%$ of the incident laser beam intensity while the rest will be delivered to the sample's surface. This negligible value clearly justifies the aforementioned assumption.

Therefore, the particles-beam interactions were ignored in this simulation to allow the heating of the underlying layers. By comparing the snapshots at $t=8 \mathrm{~ns}$ and $t=10 \mathrm{~ns}$, the bottom layer showed a higher temperature which can be clearly seen in a darker red colour after the preceding layer had left the surface. At the end of the pulse, the surface temperature drops gradually over time until a second pulse starts again and the heating cycle is repeated.

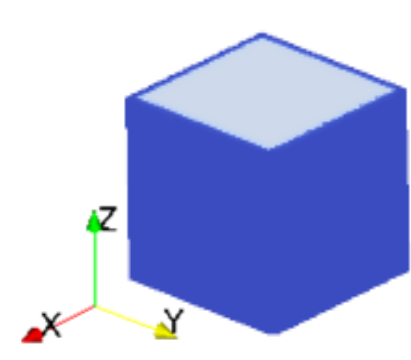

$\mathrm{t}=0.5 \mathrm{~ns}$

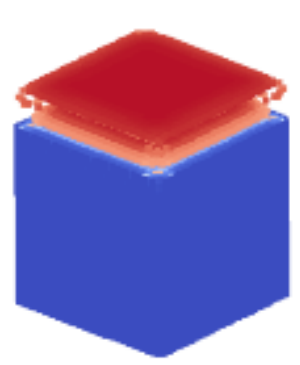

$\mathrm{t}=5.5 \mathrm{~ns}$

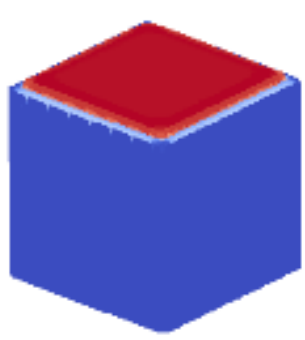

$\mathrm{t}=1.5 \mathrm{~ns}$

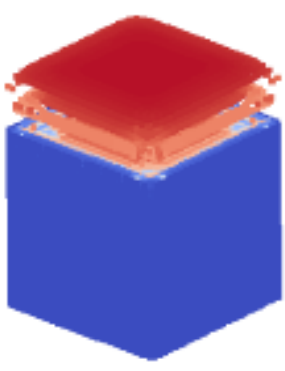

$\mathrm{t}=\mathbf{8} \mathrm{ns}$

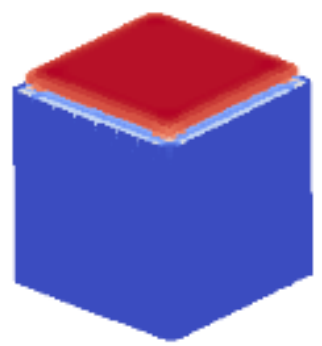

$\mathrm{t}=\mathbf{2 . 5} \mathrm{ns}$

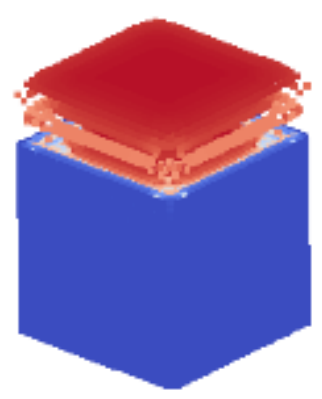

$\mathrm{t}=10 \mathrm{~ns}$

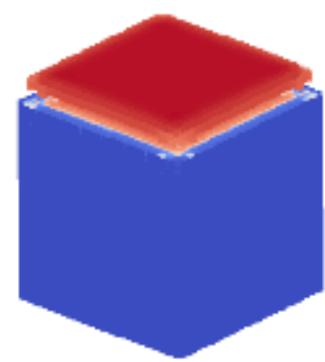

$\mathrm{t}=\mathbf{3 . 5} \mathrm{ns}$

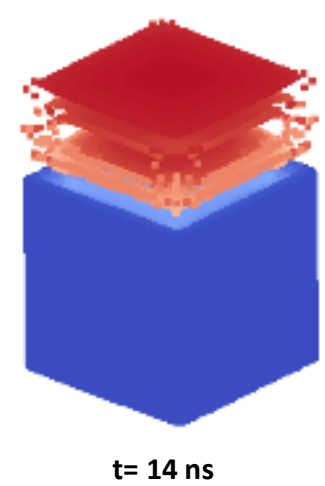

Temperature (K)

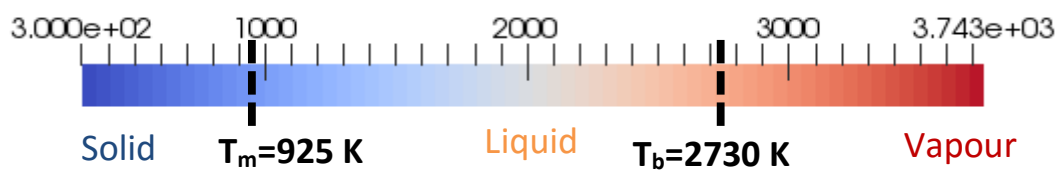

Vapour Velocity (m/s)

$\begin{array}{lllll}0.000 e+00 & 100 & 200 & 300 & 4.452 e+02\end{array}$

$|+11111| 111111111|111111111| 111111111+1111$

Figure 18 3-D view of the ablated surface showing the temperature evolution and phase change with time. Particles are ejected within $1.5 \mathrm{~ns}$ time (single shot at $10 \mathrm{~J} / \mathrm{cm}^{2}$ and $10 \mathrm{~ns}$ pulse duration). 
In order to determine the ablation depth, a vertical slice in the Z-Y plane of thickness $2 \Delta x$ is shown in Figure 19 to display the ablation depth. The surface particles were ejected within $1.5 \mathrm{~ns}$ when their temperature exceeded the boiling threshold leaving a $0.2 \mu \mathrm{m}$ crater at the top surface. Once the particles become distant from the surface ( $\nabla . r$ is greater than 2.4), the heating of the next layer begins until reaching the boiling temperature where the ejection process is repeated. The particles located at the inclined edges are ejected normal to the inclined surface reproducing a similar behaviour to that observed in the real experiments [71]. As mentioned previously in section 3.3, a criterion of ( $\nabla . r<$ 2.4) is used to identify the surface particles and the vapour velocity components are calculated according to the normal vector components in all three directions.

At the end of the pulse and when the free surface starts to cool towards the ambient temperature, the taper effect that is usually associated with the ablation process becomes evident at each edge of the crater, leaving a concave shape on the surface.
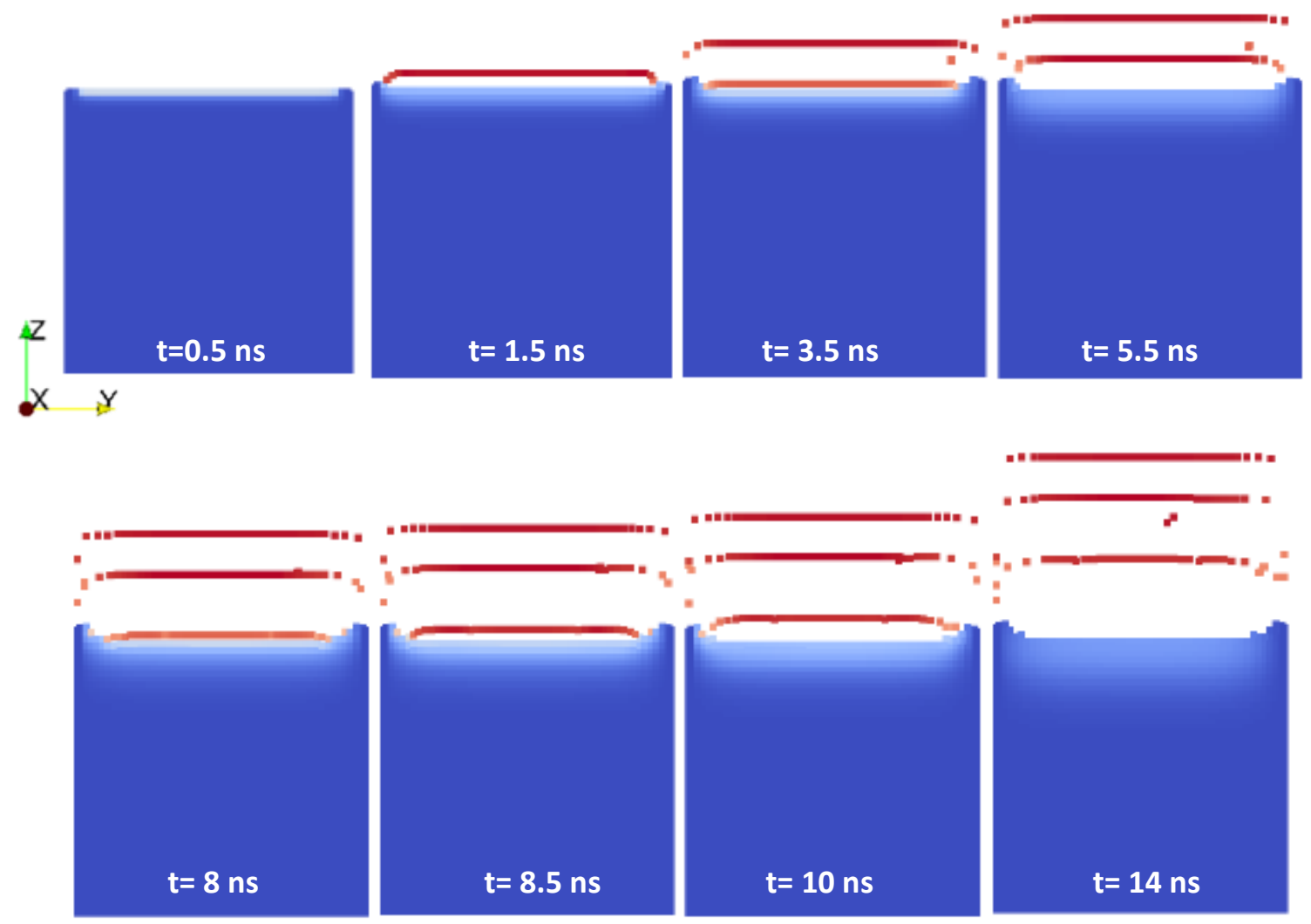

Temperature (K)

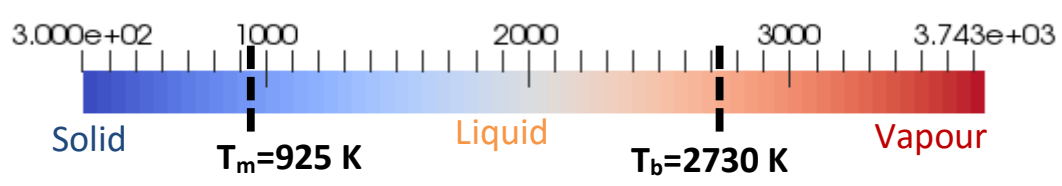

Figure 19 Cross section of the aluminium sample showing the ablation with taper effect (using single shot at $10 \mathrm{~J} / \mathrm{cm}^{2}$ and $10 \mathrm{~ns}$ pulse duration) 
The ablation depth predicted by the SPH simulation was found to be "0.6 $\mu \mathrm{m}$ ", which correlates satisfactorily with the reported value by Lutey et al., namely " $0.8 \mu \mathrm{m}$ ". The small discrepancy can be due to the different conditions of the actual sample which have not been explained by the authors in comparison with the conditions assumed in the SPH model. In reality, most aluminium surfaces suffer from oxidation. This oxidation phenomenon; however, was not taken into consideration in the SPH model. Moreover, the surface topography (roughness) of the actual sample may promote more laser absorbance than the flat surface assumed in the model.

\subsubsection{Temperature and Vapour Pressure.}

To track the change in the thermo-physical quantities with time, an SPH particle at the centre of the free surface was selected to plot its temperature evolution with time until it loses its connection with the surface. Within region I in Figure 20, the particle's temperature increases gradually with time and the heat generated by the laser is transferred into the bulk material due to conduction. The temperature then exceeds the melting point at about $t=150 \mathrm{ps}$ and the boiling temperature at $t=1 \mathrm{~ns}$ where region II starts.

In the second region, the particle starts to gain velocity due to the recoil pressure that pushes the vapour particle away from the surface. Considering the small time step (50 ps) at which all the physical quantities and the particles' coordinates are being calculated, the particle during phase II travels a very small distance within which the particle is still considered as part of the surface. Therefore, despite the fact that the particle starts to leave the surface at this stage, the particle still belongs to the surface and hence it receives more energy from the laser beam until it abandons the surface completely. This is also consistent with the condition $(\nabla \cdot r<2.4)$ that is used to identify surface particles.

After losing the connection with the lower layers, the particle's temperature begins to decrease as it becomes transparent to the laser light (see Figure 19 at $t=1.5 \mathrm{~ns}$ ). Additionally, the particle gives up some of its heat to the adjacent particles until it becomes isolated along with ejected particles of the same temperature, and this is when the temperature stabilises with time as shown in region III. This happens because the temperature gradient for a particle surrounded by particles of the same temperature will equal to zero and no heat exchange will take place between those particles. 


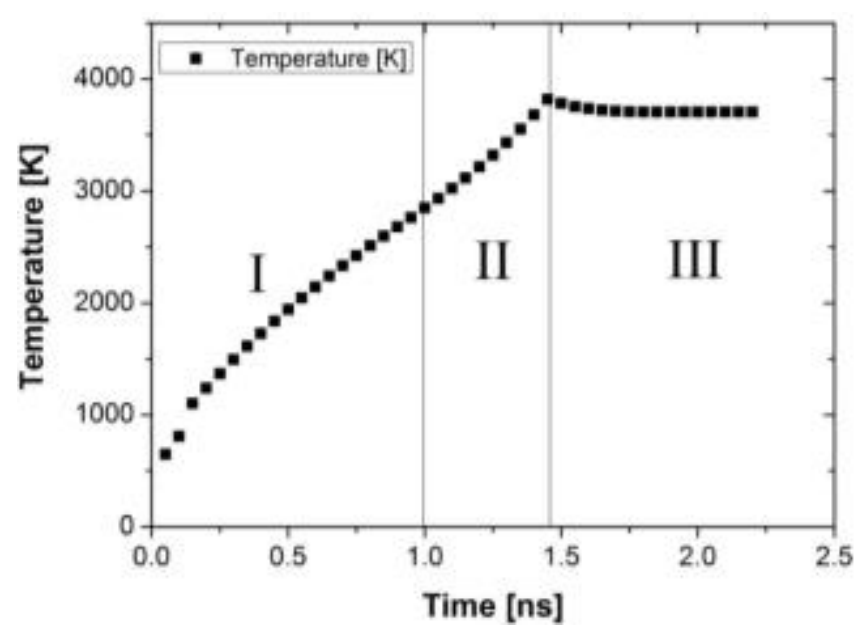

Figure 20 Temperature change with time for a surface particle obtained by SPH model (Region I: conduction heating, Region II: further heating, Region III: partial cooling after ejection)

The temperature profile is very important since it controls the ejection process and the vapour pressure associated with it. The vapour pressure values can be calculated using equation (5) with the following parametric values: $P_{a t m}=101.325 \times 10^{3}[\mathrm{~Pa}], L_{v}=10.53 \times 10^{6}[\mathrm{~J} / \mathrm{kg}], R=308.17[\mathrm{~J} / \mathrm{K} . \mathrm{Kg}]$.

Figure 21 depicts the recoil pressure values with temperature in the range between the boiling point, at which the vapour begins to form, and the maximum temperature obtained in the simulation's results. The depicted values were calculated using equation (5) according to the particle's temperature. The high recoil pressure values which reached up to 35 bar indicate that the erupted vapour is capable of leaving the surface without any help by the assist gases that are usually necessary for laser cutting and drilling processes. In the ablation processes (especially laser cleaning), a fume extraction unit is typically used to remove the ablated material so that it does not fall back to the adjacent cleaned/ablated surfaces; thus, the extraction effect does not contribute to the material ejection during the process but only to keep the vapour away from the substrate. 


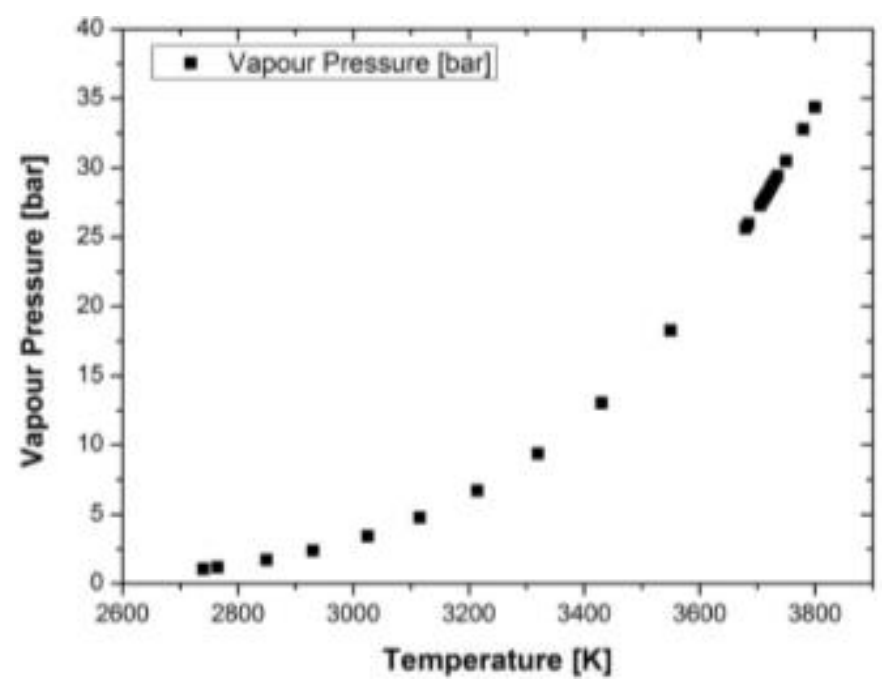

Figure $21 \mathrm{SPH}$ values of vapour pressure for surface particles a function of temperature

\subsubsection{Vapour Velocity.}

As mentioned in Section 1, finite element (FE) simulations of laser drilling, cutting, and ablation are unable to produce information on the ejected material, its behaviour and its interaction with the surrounding environment. Deleting the elements whose temperatures exceed the boiling point will not count for the interaction between the expelled particles and the solid walls of the crater, which may result in an inaccurate prediction of the process outcome. However, the Lagrangian nature of SPH makes it possible for predicting the non-linear behaviour of the physical quantities that belong to a particle wherever it travels within the domain of study.

Previous works on laser cutting/drilling calculated the recoil pressure using the Clausius-Clapeyron, which was then fed into the melt ejection velocity that was derived from Bernoulli's equation after a number of simplifying assumptions. Although the melt ejection velocity should be only assigned to the molten ejected material, the calculated values were assigned to all ejected particles regardless their temperature and phase type. Moreover, some authors [27] claimed that the recoil pressure effect becomes predominant in laser cutting due to the vapour particles build-up in the kerf; despite that, Bernoulli's equation was still being used to describe the vapour velocity although it is not the correct formulation to be used in such cases. This produces inaccurate results in the ejected material behaviour and velocity since the vapour velocity can be one or two orders of magnitude larger than the velocity calculated using Bernoulli's equation. 


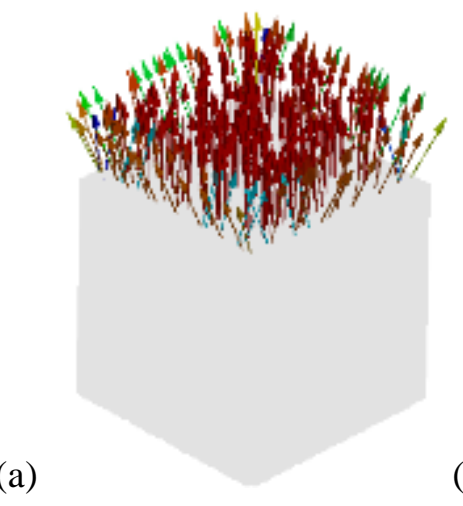

(b)

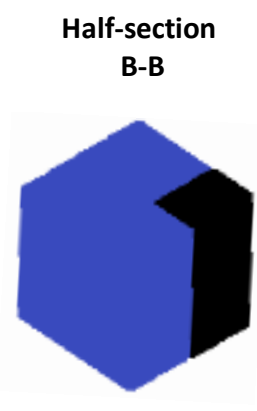

(c)
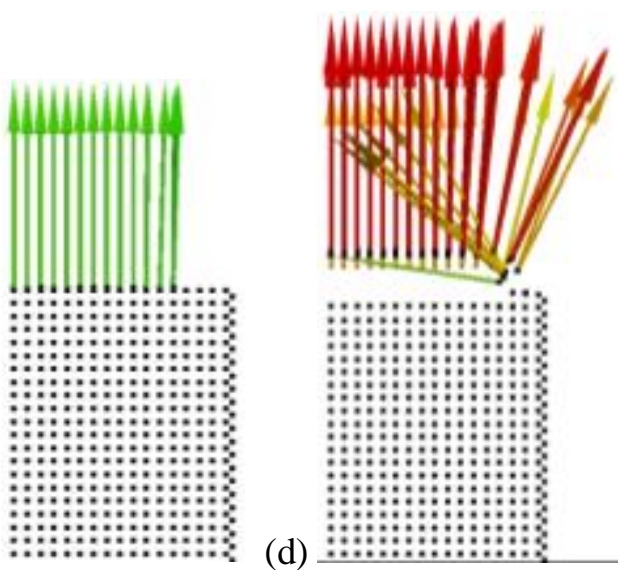

Vapour Velocity $(\mathrm{m} / \mathrm{s})$

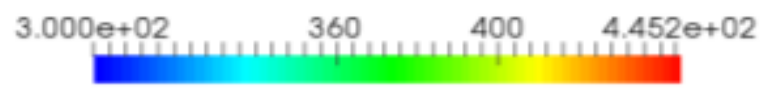

Figure 22 Vapour velocity vectors at the workpiece surface (a) 3-D view at $2 \mathrm{~ns}$ (b) half-section to be considered in the following subfigures (c) cross section B-B at $1 \mathrm{~ns}(\mathrm{~d})$ cross section B-B at $3.0 \mathrm{~ns}$

Figure 22 (a) shows the velocity vectors of the vapour particles after reaching the boiling point and how the vectors are normal to the top surface of a magnitude of about $380 \mathrm{~m} / \mathrm{s}$. In order to have a clearer view of the velocity vectors, a small section of the studied domain was isolated and projected on the front plane as shown in Figure 22 (b, c and d).

Due to the slight decrease in the particles' temperature at the edges of the domain, these particles will have slightly lower speed than those closer to the centre and their speed vectors will be slightly inclined outwards as shown in Figure 22 ( $b$ and c). Consistent with the normal-to-surface condition, particles that belong to the tapered surfaces will have their velocity vector inclined towards the inside of the domain as seen in Figure 22 (b).

Once the particle is ejected and becomes transparent to the beam, its temperature decreases significantly causing its velocity to drop, hence the ejected material will accumulate close to the surface and block any new material from being removed. This has been resolved by specifying that all ejected particles maintain a constant speed until the end of the simulation or leaving the domain. This serves two purposes: firstly, this will prevent the aggregation of removed particles above the surface and continues to allow the direct line of action of the laser onto the lower particles to be heated and removed. Secondly, this condition realistically resembles the vacuum effect in the real application which sucks all removed material away from the ablated surface.

Figure 23 depicts the evolution of velocity of a surface particle with time. It can be seen that at $1 \mathrm{~ns}$ (in Region II) the particle's temperature passes through the boiling point and the particle starts to gain 
a speed of about $380 \mathrm{~m} / \mathrm{s}$. This velocity increases gradually with the rising temperature in Region III to reach up to $450 \mathrm{~m} / \mathrm{s}$ at the end of this stage. Afterwards, the particle leaves the surface completely and maintains its velocity during the rest of the simulation time (Region IV).

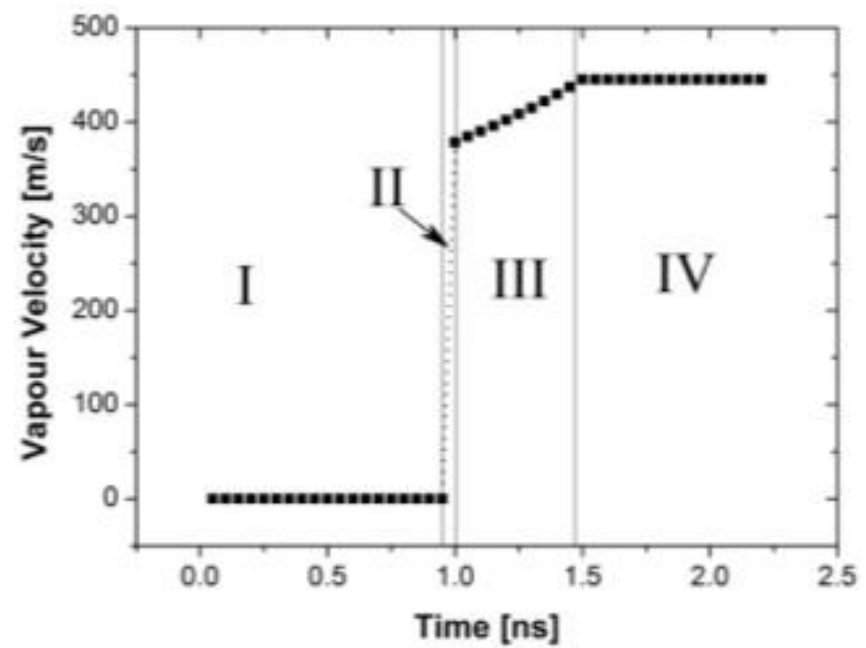

Figure 23 SPH results for the ejected particles' velocity with time (Region I: stationary state, Region II: instant ejection, Region III: velocity change with temperature, Region IV: stable speed)

According to Tam et al. [72], the particle can be ejected without melting the surface if it has an ejection acceleration greater than $\left(10^{10} \mathrm{~cm} / \mathrm{s}^{2}\right)$, that is much greater than gravitational acceleration. Taking the average increase in velocity during phase III over one time step, the SPH particles' acceleration will be about $1.4 \times 10^{11} \mathrm{~m} / \mathrm{s}^{2}$, which is greater than the threshold given by Tam et al. experimentally.

Due to the lack of experimental data on the vapour velocity of aluminium during laser ablation, the very few modelling results reported in the literature can be considered for comparison. Hamadi et al. [73] created a finite volume model using "Fluent" code to estimate the vapour velocity of aluminium when ablated with nanosecond UV laser beam. It was assumed that the ambient pressure is $10^{2} \mathrm{~Pa}$ and the aluminium target was irradiated with a $25 \mathrm{~ns}$ pulse. Their results showed that the maximum particle velocity was about $1110 \mathrm{~m} / \mathrm{s}$ at the centre of the ablated area, and it reduces to $167 \mathrm{~m} / \mathrm{s}$ away from the centre, with an average speed of about $640 \mathrm{~m} / \mathrm{s}$. This average value is in good agreement with the SPH results obtained from this work taking into consideration the different boundary conditions of the two models. Moreover, the authors indicated that the vapour velocity reduces when the ambient pressure increases, and taking into account that the ambient pressure in this work is $10^{5}$ $\mathrm{Pa}$, the SPH particles velocity is then expected to be lower than the value reported by Hamadi et al.

Rajendran et al. [74] studied a similar test case using a numerical model based on the kinetic description of the Knudsen layer. Their numerical results, which according to the authors showed a 
good agreement with other analytical models, showed that the maximum velocity reached up to 750 $\mathrm{m} / \mathrm{s}$ after $15 \mathrm{~ns}$ at the surface of the target. These values indicate that SPH prediction of the vapour velocity lies within a satisfactory range of values for the considered model and material.

\subsubsection{Further Validation of Laser Ablation Depth.}

After considering one set of parameters to validate the SPH model, a wider range of fluences and pulse durations are tested to further validate the behaviour of the proposed model.

Lutey et al. [69] conducted experiments using nanosecond laser with $10 \mathrm{~ns}$ pulse duration, $30 \mathrm{kHz}$ repetition rate and beam fluence of $4-20 \mathrm{~J} / \mathrm{cm}^{2}$. A so-called "unidimensional" numerical model was created to predict the ablation depth of the studied aluminium sheet. Figure 24 shows a good agreement in the predicted ablation depth between the SPH results, the experimental and numerical data by Lutey et al. at different fluence values, achieving about $1.0-1.2 \mu \mathrm{m} /$ pulse at $20 \mathrm{~J} / \mathrm{cm}^{2}$.

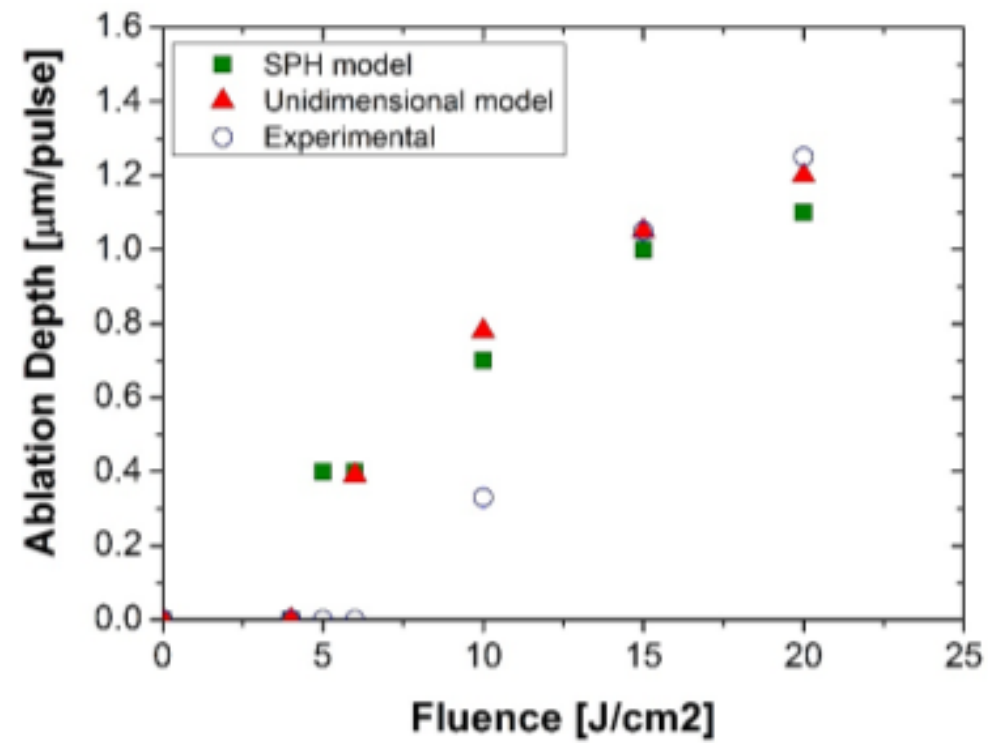

Figure 24 Ablation depths at different fluences $4-23 \mathrm{~J} / \mathrm{cm}^{2}$ using $10 \mathrm{~ns}$ pulse with $30 \mathrm{kHz}$ repetition rate. SPH results are compared with experimental and numerical values reported in Lutey et al.

From Figure 24, the difference between the SPH modelling results and Lutey's numerical data can be attributed to the fact that their unidimensional model only calculates the heat conduction in one direction without taking the heat losses into account, while the 3-D nature of the SPH model accounts for the heat flow in the other two directions of the domain and for the convection and radiation losses at the sample's free surface. The discrepancy between the experimental and the simulation results can be explained by the fact that the experimental values of the ablation depth per pulse were taken as the average of the total depth over the aggregate number of pulses. Additionally, the deep ablated surfaces during the experiments enhance the internal reflection of the laser beam on the side walls of the hole 
and promote more absorption of the beam in comparison with the single-shot ablation achieved in the simulation. Furthermore, the surface conditions of the sample during the experiments may differ from those in the simulation since there was no mentioning of such information by the authors. For instance, oxidation layers usually form on the free surface of aluminium and their low thermal conductivity $(35 \mathrm{~W} / \mathrm{mK})$ and the greater density $\left(3750 \mathrm{~kg} / \mathrm{m}^{3}\right)$ makes it more difficult to be ablated in comparison with pure aluminium.

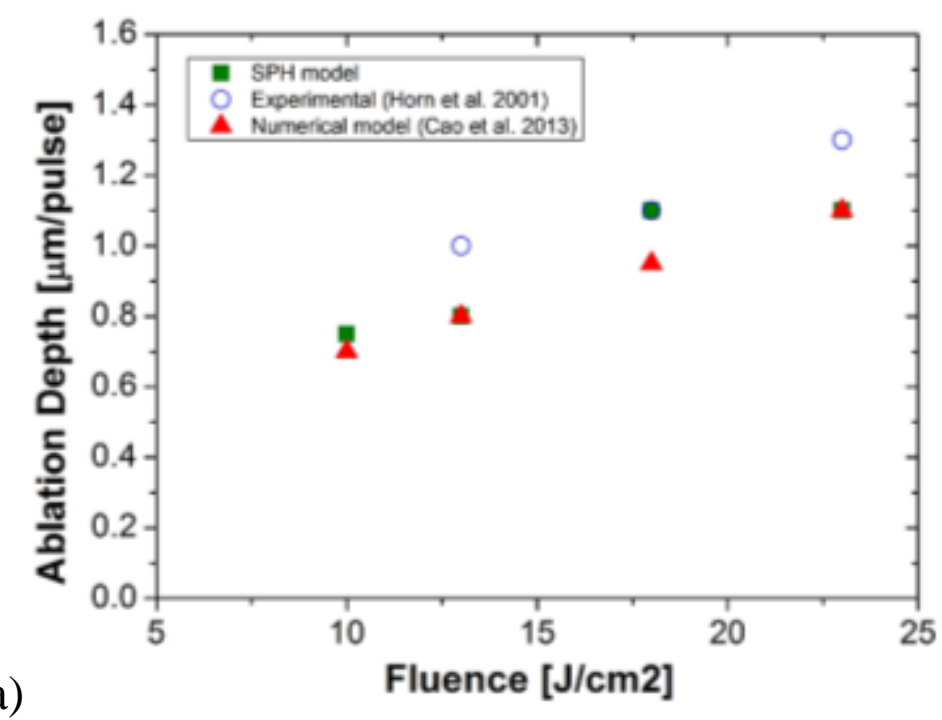

(a)

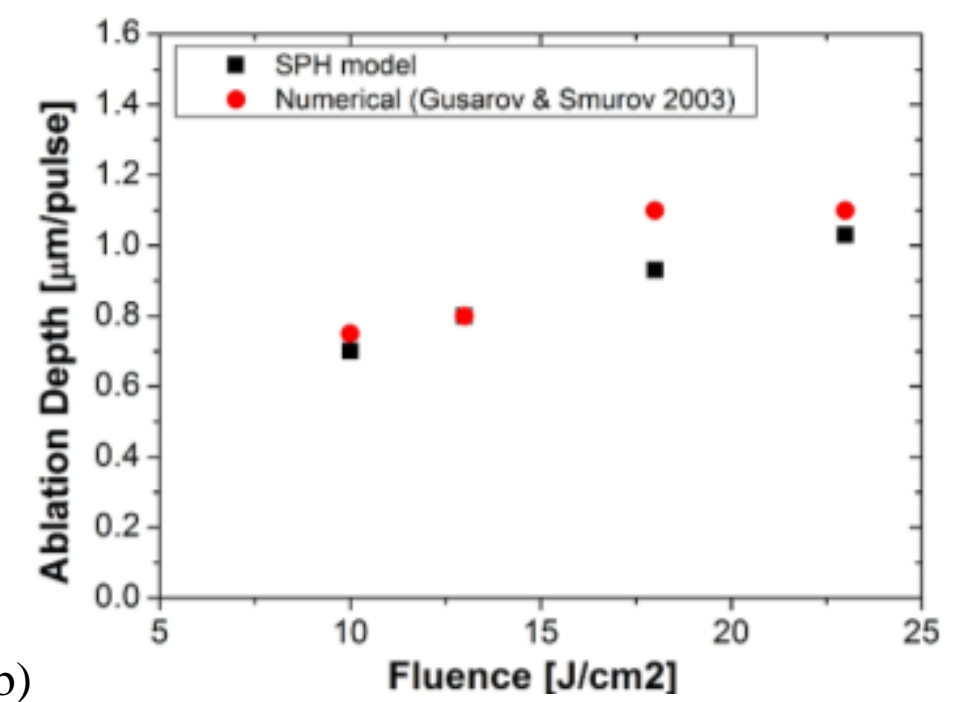

Figure $25 \mathrm{SPH}$ results of Ablation depth compared to literature experimental and numerical data at different fluences $4-23 \mathrm{~J} / \mathrm{cm}^{2}$ using 6 ns pulse.

Figure 25 (a) shows a different case created using shorter pulse durations of 6 ns and laser fluences 10-25 J/ $\mathrm{cm}^{2}$ on an aluminium target. In this case, the SPH results were compared with both experimental and numerical data from different sources [71, 75]. As mentioned previously, all 
experimental data are averaged over the total number of pulses ( 400 pulses in this case) in order to obtain the ablation depth per pulse.

In Figure 25 (a), a good correlation between the SPH prediction and the reported data can be noted with a linear increase of the ablation depth with the energy density. Despite that, the experimental work has reported higher ablation rates compared to the simulations over the studied fluence regime. This can be attributed to the so-called "incubation effect" that takes place during multi-pulses laser ablation as well as the different surface conditions on the actual samples [76].

The same SPH results also achieved a good correlation with the ablation rates predicted by a numerical model [77] of supercritical ablation with an optical breakdown in the volume of the vapour phase (see Figure $25 \mathrm{~b}$ ). This model was different from the previous numerical models as it includes the interaction between the laser radiation and the gas phase produced when the vapour temperature exceeds the critical temperature of aluminium.

The good agreement with both the experimental work and the different simulation models indicates that the thermal ablation mechanism can be applied successfully to predict the ablation depth in the low to medium fluence range, and the SPH model was able to not only predict the ablation depth but also to give an insight into the vapour phase and its characteristics.

\section{Conclusions}

A 3-D SPH model has been presented to predict the characteristics of the laser ablation process after evaluating the heat conduction behaviour in such processes, as well as the need and the sensitivity of the smoothing kernel correction at the free surfaces in SPH modelling. According to the results reported in this work, the following conclusions can be drawn:

- The proposed model in this paper showed an excellent agreement with the analytical solution using different levels of power intensity and surface reflectivity and produced credible temperature values which were then utilised in laser ablation simulation.

- KGC and Schwaiger correction were able to correct the deviation caused by the truncated support at the domain borders for the gradient and Laplacian of the studied functions respectively. However, for non-linear functions such as hyperbolic and logarithmic functions both correction methods were only able to reduce the deviation at the boundaries without matching the analytical solution.

- SPH predictions of the ablation depth for different process parameters were in a good agreement with both experimental and numerical data reported in the literature. Unlike other mesh-based methods in which no information can be provided on the ejected material, the SPH model was not only able to provide the temperature and velocity of the ejected particles, 
but also the effect of the interaction between them as well as the direction and the pattern of the ejection. 


\section{Appendix}

The free surface on the top of the specimen in the SPH model simulates the adiabatic condition in which no heat exchange occurs due to convection or radiation with the surrounding. Hence, pure heat conduction behaviour of the SPH particles can be observed and compared to the 1-D analytical solution of the heat conduction PDE. Assuming that the material is initially at the room temperature $300^{\circ} \mathrm{K}$ when $t=0$ and that the temperature at $z=\infty$ at $t>0$ is kept at the room temperature, the heating and cooling phases for a single laser pulse can be described using the following equations [62]:

$$
\begin{gathered}
\left.T(z, t)\right|_{t<t_{p}}=T_{0}+\frac{2 I_{0}(1-R)}{k} \sqrt{D \cdot t}\left(\operatorname{ierfc}\left[\frac{z}{2 \sqrt{D . t}}\right]\right) \\
\left.T(z, t)\right|_{t>t_{p}}=T_{0}+\frac{2 I_{0}(1-R)}{k}\left(\sqrt{D . t} \operatorname{ierfc}\left[\frac{z}{2 \sqrt{D . t}}\right]-\sqrt{D \cdot\left(t-t_{p}\right)} \operatorname{ierfc}\left[\frac{z}{2 \sqrt{D \cdot\left(t-t_{p}\right)}}\right]\right)
\end{gathered}
$$

where $z$ is the depth at which the temperature is calculated, $T_{0}$ is the initial temperature, $I_{0}$ is the Laser intensity, $R$ is the surface reflectivity, $t_{p}$ is the pulse duration, and $t$ is time.

If the laser beam diameter is of the same order of the thermal penetration given by $(z \cdot D)^{0.5}$, then the heat conduction in the radial direction should be taken into account and the beam diameter $a$ should be included in the analytical solution. Otherwise, the terms containing the parameter $a$ should be ignored.

Taking into account the aforementioned notes, a corrected form of the analytical solution produced by Nath et al. [62] is now applied as two sets of equations to describe the heating and the cooling phases.

During the heating phase (laser-ON), the temperature at depth $z$ is given by: 


$$
\begin{aligned}
& \left.T(z, t)\right|_{t<t_{p}}=T_{0}+\frac{2 I_{0}(1-R) \sqrt{D}}{k}\left\{\sqrt { t - ( N - 1 ) ( t _ { p } + t _ { r } ) } \left(\operatorname{ierfc}\left[\frac{z}{2 \sqrt{D\left(t-(N-1)\left(t_{p}+t_{r}\right)\right)}}\right]\right.\right. \\
& \left.-\operatorname{ierfc}\left[\frac{\sqrt{z^{2}+a^{2}}}{2 \sqrt{D\left(t-(N-1)\left(t_{p}+t_{r}\right)\right)}}\right]\right) \\
& +\sum_{n=1}^{N-1}\left\{\sqrt { t - ( n - 1 ) ( t _ { p } + t _ { r } ) } \left(\operatorname{ierfc}\left[\frac{z}{2 \sqrt{D\left(t-(n-1)\left(t_{p}+t_{r}\right)\right)}}\right]\right.\right. \\
& \left.-\operatorname{ierfc}\left[\frac{\sqrt{z^{2}+a^{2}}}{2 \sqrt{D\left(t-(n-1)\left(t_{p}+t_{r}\right)\right)}}\right]\right) \\
& -\sqrt{t-\left(n t_{p}+(n-1) t_{r}\right)}\left(\operatorname{ierfc}\left[\frac{z}{2 \sqrt{D\left(t-\left(n t_{p}+(n-1) t_{r}\right)\right)}}\right]\right. \\
& \left.\left.\left.-\operatorname{ierfc}\left[\frac{\sqrt{z^{2}+a^{2}}}{2 \sqrt{D\left(t-\left(n t_{p}+(n-1) t_{r}\right)\right)}}\right]\right)\right\} \quad\right\}
\end{aligned}
$$

where $a$ is the beam diameter, $N$ is the total number of pulses, $n$ is the pulse number, $t_{p}$ is the pulse duration and $t_{r}$ is the relaxation time. During the cooling phase (laser-OFF), the temperature at $z$ depth is given by:

$$
\begin{aligned}
& \left.T(z, t)\right|_{t>t_{p}}=T_{0}+\frac{2 I_{0}(1-R) \sqrt{D}}{k}\left\{\sqrt{t}\left(\operatorname{ierfc}\left[\frac{z}{2 \sqrt{D(t)}}\right]-\operatorname{ierfc}\left[\frac{\sqrt{z^{2}+a^{2}}}{2 \sqrt{D(t)}}\right]\right)\right. \\
& -\sqrt{t-t_{p}}\left(\operatorname{ierfc}\left[\frac{z}{2 \sqrt{D\left(t-t_{p}\right)}}\right]-\operatorname{ierfc}\left[\frac{\sqrt{z^{2}+a^{2}}}{2 \sqrt{D\left(t-t_{p}\right)}}\right]\right) \\
& +\sum_{n=1}^{N-1}\left\{\sqrt{t-n\left(t_{p}+t_{r}\right)}\left(\operatorname{ierfc}\left[\frac{z}{2 \sqrt{D\left(t-n\left(t_{p}+t_{r}\right)\right)}}\right]-\operatorname{ierfc}\left[\frac{\sqrt{z^{2}+a^{2}}}{2 \sqrt{D\left(t-n\left(t_{p}+t_{r}\right)\right)}}\right]\right)\right. \\
& -\sqrt{t-\left((n+1) t_{p}+n t_{r}\right)}\left(\operatorname{ierfc}\left[\frac{z}{2 \sqrt{D\left(t-\left((n+1) t_{p}+n t_{r}\right)\right)}}\right]\right. \\
& \left.\left.\left.-\operatorname{ierfc}\left[\frac{\sqrt{z^{2}+a^{2}}}{2 \sqrt{D\left(t-\left((n+1) t_{p}+n t_{r}\right)\right)}}\right]\right)\right\}\right\}
\end{aligned}
$$




\section{References:}

[1] Feliu Jr. S., Pardo A., Merino M. C., Coy A. E., Viejo F., A. R., Correlation between the surface chemistry and the atmospheric corrosion of AZ31, AZ80 and AZ91D magnesium alloys, Appl Surf Science, 255 (2009).

[2] Yong-Feng L., Wen-Dong S., Teck-Seng L., Laser cleaning of micro-particles from a solid surface --theory and applications, Materials Chemistry and Physics, 51 (1998) 181-185.

[3] A.W. AlShaer, L. Li, A. Mistry, The effects of short pulse laser surface cleaning on porosity formation and reduction in laser welding of aluminium alloy for automotive component manufacture, Optics \& Laser Technology, 64 (2014) 162-171.

[4] M.W. Turner, P.L. Crouse, L. Li, Comparison of mechanisms and effects of Nd:YAG and CO2 laser cleaning of titanium alloys, Applied Surface Science, 252 (2006) 4792-4797.

[5] Y.W. Zheng, B.S. Luk'yanchuk, Y.F. Lu, W.D. Song, Z.H. Mai, Dry laser cleaning of particles from solid substrates: Experiments and theory, Journal of Applied Physics, 90 (2001) 2135-2142.

[6] Y.F. Lu, W.D. Song, Y. Zhang, M.H. Hong, T.C. Chong, Laser removal of particles from solid surfaces, RIKEN Rev, 32 (2001) 64-70.

[7] W.D. Song, Y.F. Lu, M.H. Hong, T.C. Chong, T.S. Low, Theoretical and experimental investigation on laser dry cleaning of particles from substrate surfaces, in: Advanced Applications of Lasers in Materials Processing/Broadband Optical Networks/Smart Pixels/Optical MEMs and Their Applications. IEEE/LEOS 1996 Summer Topical Meetings:, 1996, pp. 69-70.

[8] R. Oltra, O. Yavaş, F. Cruz, J.P. Boquillon, C. Sartori, Modelling and diagnostic of pulsed laser cleaning of oxidized metallic surfaces, Applied Surface Science, 96-98 (1996) 484-490.

[9] Bloisi F, Di Blasio G, Vicari L, Zoncheddu M, One-dimensional modelling of 'verso' laser cleaning, J. Mod. Optics, 53 (2006) 1121-1129.

[10] J. Marczak, K. Jach, A. Sarzyński, Numerical Modelling of Laser Cleaning and Conservation of Artworks, in: K. Dickmann, C. Fotakis, J. Asmus (Eds.) Lasers in the Conservation of Artworks, Springer Berlin Heidelberg, 2005, pp. 319-326.

[11] J.D. Kelley, M.I. Stuff, F.E. Hovis, G.J. Linford, Removal of small particles from surfaces by pulsed laser irradiation: observations and a mechanism, in, 1991, pp. 211-219.

[12] S. Kudryashov, S.D. Allen, Experimental and Theoretical Studies of Laser Cleaning Mechanisms for Submicrometer Particulates on Si Surfaces, Particulate Science and Technology, 24 (2006) 281299.

[13] B.S. Luk'yanchuk, N. Arnold, S.M. Huang, Z.B. Wang, M.H. Hong, Three-dimensional effects in dry laser cleaning, Applied Physics A, 77 (2003) 209-215.

[14] N. Arnold, Theoretical description of dry laser cleaning, Appl. Surf. Sci., 208 (2003) 15-22.

[15] N. Arnold, G. Schrems, T. Mühlberger, M. Bertsch, M. Mosbacher, P. Leiderer, D. Bäuerle, Dynamic Particle Removal by Nanosecond Dry Laser Cleaning: Theory, in: I.M.Y.F.L.K.S.J.J. Dubowski (Ed.) Second International Symposium on Laser Precision Microfabrication, Proceedings of SPIE Singapore, Singapore, 2001, pp. 340-346.

[16] M. Colina , C. Molpeceres, M. Morales, F. Aliens-Perkins, G. Guadaño, J. L Ocaña Laser ablation modelling of aluminium, silver and crystalline silicon for applications in photovoltaic technologies, Surf. Eng., 27 (2011) 414-423.

[17] D. Autrique, V. Alexiades, H. Khanal, HYDRODYNAMIC MODELING OF ns LASER ABLATION, in: Ninth MSU-UAB Conference on Differential Equations and Computational Simulations, Electronic Journal of Differential Equations, 2013, pp. 1-14.

[18] N. Bityurin, B. S. Luk'yanchuk, M. H. Hong, T. C. Chong, Models for Laser Ablation of Polymers, Chem. Rev, 103, (2003).

[19] M. Arronte, P. Neves, R. Vilar, Modelling of laser cleaning of metallic particles on silicon substrates, in: Lasers and Electro-Optics Europe, 2003. CLEO/Europe. 2003 Conference on, 2003, pp. 561. 
[20] Y. Liyang, W. Zengbo, L. Lin, Multiphysics modelling and simulation of dry laser cleaning of micro-slots with particle contaminants, Journal of Physics D: Applied Physics, 45 (2012) 135401. [21] Lutey H. A. , An improved model for nanosecond pulsed laser ablation of metals, Journal of Applied Physics 114 (2013) 083108-083110.

[22] Marimuthu S. , Mhich A. , Molchan I. S. , Whitehead D. , Wang Z. B. , Mativenga P., Li L. , Liu Z. , Grafton-Reed C. , Cheetham S. , Dilworth S. , Numerical Simulation of Excimer Laser Cleaning of Film and Particle Contaminants, Journal of Heat Transfer, 135 (2013) 121301-121301.

[23] Shalahim N. S. M., Mon T. T., Ismail M. F., Rashid M. F. F., Rejab M.R.M., Finite element simulation of laser-micromachining, in: Proc. International MultiConference of Engineers and Computers Scientists (IMECS), Hong Kong, 2010.

[24] Gross M., Smooth particle hydrodynamics (SPH) modelling of laser cutting, in: Int. Congress on Applications of Lasers \& Electro-Optics (ICALEO), University of Cambridge; Cambridge Great Britain., 2008 pp. 637-644.

[25] Liu G. R., Liu M. B., Smoothed particle hydrodynamics: a meshfree particle method, World Scientific, New Jersey, 2003.

[26] Monaghan J.J., Smoothed particle hydrodynamics, Rep. Prog. Phys., 68 (2005) 1703-1759.

[27] Muhammad N., B. D. Rogers, L. Li, Understanding the behaviour of pulsed laser dry and wet micromachining processes by multi-phase smoothed particle hydrodynamics (SPH) modelling, J.

Phys. D: Appl. Phys., 46 ( 2013 ) 095101

[28] Lucy L. B., A numerical approach to the testing of the fission hypothesis, Astronom J., 82 (1977) 1013-1024.

[29] Gingold R. A., Monaghan J. J., Smoothed particle hydrodynamics-theory and application to nonspherical stars, Mon. Not. R. Astronom. Soc., 181 (1977) 375-389.

[30] R.A. Dalrymple, B.D. Rogers, Numerical modeling of water waves with the SPH method, Coastal Engineering, 53 (2006) 141-147.

[31] Rogers B D , Dalrymple R A SPH modeling of Tsunami waves, Advances in Coastal and Ocean Engineering , Advanced Numerical Models for Tsunami Waves and Runup (Singapore: World Scientific), 10 (2008).

[32] Rogers B D, Dalrymple R A, Gesteria M , Knio O, Smoothed particle hydrodynamics for naval hydrodynamics, in: A.H.C.a.P. Ferrant (Ed.) Proc 18th Int Workshop on Water Waves and Floating Bodies Nantes, France, 2003.

[33] Cleary P W, Prakash M, Ha J, Novel applications of smoothed particle hydrodynamics (SPH) in metal forming, J. Mater. Process. Technol. , 177 (2006) 41-48.

[34] Zhang $M$, Zhang $H$, Zheng L, Application of smoothed particle hydrodynamics method to free surface and solidification problems Numer. Heat Transfer A., 52 (2007) 299-314.

[35] Xiong H-B , Zhu J Study of droplet deformation, heat conduction and solidification using incompressible smoothed particle hydrodynamics method, in: 9th Int. Conf. on Hydrodynamics, Shanghai, China, 2010.

[36] C. Demuth, M. Bieda, A.F. Lasagni, A. Mahrle, A. Wetzig, E. Beyer, Thermal simulation of pulsed direct laser interference patterning of metallic substrates using the smoothed particle hydrodynamics approach, Journal of Materials Processing Technology, 212 (2012) 689-699.

[37] M. Tong, D.J. Browne, Smoothed particle hydrodynamics modelling of the fluid flow and heat transfer in the weld pool during laser spot welding, Mater. Sci. Eng., 27 (2011) 1-7.

[38] Y. Yan, L. Li, K. Sezer, W. Wang, D. Whitehead, L. Ji, Y. Bao, Y. Jiang, CO2 laser underwater machining of deep cavities in alumina, Journal of the European Ceramic Society, 31 (2011) 27932807.

[39] J.E.B. J. K. Chen, NUMERICAL STUDY OF ULTRASHORT LASER PULSE INTERACTIONS WITH METAL FILMS, Numerical Heat Transfer, Part A: Applications, 40 (2001) 1-20.

[40] Y. Cao, Y.C. Shin, Multi-scale modeling of phase explosion in high fluence nanosecond laser ablation and clarification of ablation depth prediction criterion, Applied Surface Science, 357, Part A (2015) 74-85. 
[41] P.W. Cleary, J.J. Monaghan, Conduction Modelling Using Smoothed Particle Hydrodynamics, Journal of Computational Physics, , 148 (1999) 227-264.

[42] J.H. Jeong, M.S. Jhon, J.S. Halow, J. van Osdol, Smoothed particle hydrodynamics: Applications to heat conduction, Computer Physics Communications, 153 (2003) 71-84.

[43] Turner M. W. , Crouse P. L. , Li L., Comparative interaction mechanisms for different laser systems with selected materials on titanium alloys, Applied Surface Science, 253 (2007) 7992-7997.

[44] D.W. Bäuerle, Laser Processing and Chemistry, Fourth Edition ed., Springer-Verlag Berlin Heidelberg, 2011.

[45] P.W. Atkins, J. De Paula, Atkins' Physical Chemistry, Oxford University Press, 2002.

[46] R. Kelly, On the dual role of the Knudsen layer and unsteady, adiabatic expansion in pulse sputtering phenomena, The Journal of Chemical Physics, 92 (1990) 5047-5056.

[47] Muhammad N., Laser micromachining of coronary stents for medical applications, in: Faculty of Engineering and Physical Sciences, The Univeristy of Manchester, manchester, 2012.

[48] Morris J.P., P.J. Fox, Y. Zhu, Modeling Low Reynolds Number Incompressible Flows Using SPH. Journal of Computational Physics, 136 (1997) 214-226.

[49] A. Mayrhofer, B.D. Rogers, D. Violeau, M. Ferrand, Investigation of wall-bounded flows using $\mathrm{SPH}$ and the unified semi-analytical wall boundary conditions, Computer Physics Communications, 184 (2013) 2515-2527.

[50] Monaghan J., Kos A., Solitary Waves on a Cretan Beach, Journal of Waterway, Port, Coastal, and Ocean Engineering, 125 (1999).

[51] M. Yildiz, R.A. Rook, A. Suleman, SPH with the multiple boundary tangent method, International Journal for Numerical Methods in Engineering, 77 (2009) 1416-1438.

[52] G. Fourtakas, R. Vacondio, B.D. Rogers, On the approximate zeroth and first-order consistency in the presence of 2-D irregular boundaries in SPH obtained by the virtual boundary particle methods, International Journal for Numerical Methods in Fluids, 78 (2015) 475-501.

[53] W. Liu, L. S., B. T., Moving least square Kernel Galerkin method (I) methodology and convergence, Comput. Methods Appl. Mech. Engineering., (1997) 143:113.

[54] Schwaiger F., An implicit corrected SPH formulation for thermal diffusion with linear free surface boundary conditions, International Journal for Numerical Methods in Engineering, 75 (2008) 647-671.

[55] Gómez-Gesteira M., Rogers B.D., Crespo A.J.C., Dalrymple R.A, Narayanaswamy M., Dominguez J.M., SPHysics - development of a free-surface fluid solver- Part 1: Theory and Formulations, Computers \& Geosciences, 48 (2012) 289-299.

[56] Monaghan J.J., Smoothed Particle Hydrodynamics, Ann. Rev. Astron. Astro, 30 (1992) 543.

[57] Liu G. R., Liu M. B., Smoothed particle hydrodynamics: a meshfree particle method, World Scientific, New Jersey, 2003.

[58] Monaghan J., Huppert H., Grae Worster M., Solidification using smoothed particle hydrodynamics, Journal of Computational Physics 206 (2005) 684-705.

[59] E.S. Lee, Truly incompressible approach for computing incompressible flow in SPH and comparisons with the traditional weakly compressible approach, in: School of Mechanical, Aerospace and Civil Engineering, The University of Manchester, 2007, pp. 165.

[60] S.J. Lind, R. Xu, P.K. Stansby, B.D. Rogers, Incompressible smoothed particle hydrodynamics for free-surface flows: A generalised diffusion-based algorithm for stability and validations for impulsive flows and propagating waves, Journal of Computational Physics, 231 (2012) 1499-1523.

[61] A.J.C. Crespo, M. Gómez-Gesteira, R. Dalrymple, Boundary Conditions Generated by Dynamic Particles in SPH Methods, Computers, Materials, \& Continua, 5 (2007) 173-184.

[62] A.K. Nath, A. Gupta, F. Benny, Theoretical and experimental study on laser surface hardening by repetitive laser pulses, Surface and Coatings Technology, 206 (2012) 2602-2615.

[63] Wolfram, L2 Norm Error, in.

[64] A.W. AlShaer, L. Li, A. Mistry, Effect of filler wire properties on porosity formation in laser welding of AC-170PX aluminium alloy for lightweight automotive component manufacture, 
Proceedings of the Institution of Mechanical Engineers, Part B: Journal of Engineering Manufacture, (2015).

[65] P.J. Roache, QUANTIFICATION OF UNCERTAINTY IN COMPUTATIONAL FLUID DYNAMICS, Annual Review of Fluid Mechanics, 29 (1997) 123-160.

[66] P.J. Roache, Perspective: A Method for uniform reporting of grid refinement studies, Journal of Fluid Engineering, 116 (1994) 405-413.

[67] X. Xu, Phase explosion and its time lag in nanosecond laser ablation, Applied Surface Science, 197-198 (2002) 61-66.

[68] G. Faussurier, C. Blancard, P.L. Silvestrelli, Evaluation of aluminum critical point using an Itextit\{ab initio\} variational approach, Physical Review B, 79 (2009) 134202.

[69] A.H.A. Lutey, M. Sozzi, S. Selleri, A. Cucinotta, P.G. Molari, Picosecond and nanosecond pulsed laser ablation of aluminium, polypropylene, polyethylene, and their thin-film combinations, in, 2013, pp. 860804-860804-860811.

[70] Hoffman J., Szymanski Z., Absorption of the laser beam during welding with CO2 laser, Optica Applicata, 32 (2002) 129-145

[71] I. Horn, M. Guillong, D. Günther, Wavelength dependant ablation rates for metals and silicate glasses using homogenized laser beam profiles - implications for LA-ICP-MS, Applied Surface Science, 182 (2001) 91-102.

[72] Tam A. C., Leung W. P., Zapka W., Ziemlich W., Laser cleaning techniques for removal of surface particulates, Journal of Applied Physics, 71 (1992) 3515.

[73] F. Hamadi, E.H. Amara, T. Kerdja, D. Mezaoui, Numerical modelling of aluminium vapour produced by nanosecond UV laser beam ablation, physica status solidi (c), 5 (2008) 3314-3318.

[74] S. Rajendran, M. Keidar, I. Boyd, Ablation and Plasma Formation Due to Laser Irradiance, in: AIAA-2007-4378, 38th AIAA Plasmadynamics and Lasers Conference In conjunction with the 16th International Conference on MHD Energy Conversion, Miami, Florida, 2007.

[75] Y. Cao, X. Zhao, Y.C. Shin, Analysis of nanosecond laser ablation of aluminum with and without phase explosion in air and water, Journal of Laser Applications, 25 (2013) 032002.

[76] S. Xiao, E. Gurevich, A. Ostendorf, Incubation effect and its influence on laser patterning of ITO thin film, Applied Physics A, 107 (2012) 333-338.

[77] A.V. Gusarov, I. Smurov, Near-surface laser-vapour coupling in nanosecond pulsed laser ablation, Journal of Physics D: Applied Physics, 36 (2003) 2962. 